Research Article

\title{
Research on Aluminum Honeycomb Buffer Device for Soft Landing on the Lunar Surface
}

\author{
Wei Wei $\mathbb{D}^{1}$, Shijie Zhang $\mathbb{D},{ }^{1}$ Ximing Zhao, ${ }^{1}$ Xinyu Quan ${ }^{D},{ }^{1}$ Jie Zhou, ${ }^{1}$ Nan Yu, \\ Hongxiang Wang, ${ }^{1}$ Meng $L i \mathbb{D}^{2}$ and Xuyan Hou $\mathbb{D}^{1}$ \\ ${ }^{1}$ State Key Laboratory of Robotics and System, Harbin Institute of Technology, No. 2 Yikuang Street, Nangang, Harbin City, \\ Heilongjiang Province 150080, China \\ ${ }^{2}$ Qian Xuesen Laboratory of Space Technology, China Academy of Space Technology, Beijing 100094, China
}

Correspondence should be addressed to Meng Li; limeng@qxslab.cn and Xuyan Hou; houxuyan@hit.edu.cn

Received 8 July 2021; Accepted 22 September 2021; Published 31 October 2021

Academic Editor: Jinyang Xu

Copyright (c) 2021 Wei Wei et al. This is an open access article distributed under the Creative Commons Attribution License, which permits unrestricted use, distribution, and reproduction in any medium, provided the original work is properly cited.

\begin{abstract}
To obtain the resources of the moon, humans have launched a series of exploration activities on the moon, and the landing buffer device is an indispensable device on the lander required to perform lunar surface exploration missions. It can effectively protect the lander during landing scientific payloads such as instruments on the lander. Based on the mechanical properties and deformation mechanism of the aluminum honeycomb as buffer material, this paper compares and analyzes different simulation schemes and finally establishes the bonding model of the honeycomb by using the discrete element method; the parameters of the honeycomb material are matched through compression experiments to verify the discrete element honeycomb simulation and the feasibility of the scheme and its parameters. To meet the buffering requirements of large landers, a spider web honeycomb structure is proposed, its modeling method is studied by using the discrete element secondary development program, and the model is compressed as a whole to verify the energy consumption characteristics of the spider web honeycomb structure. Aiming at the honeycomb buffer device during the landing process, the cobweb honeycomb buffer structure and its corresponding landing coupling model were established using the discrete element method, the landing process was simulated and analyzed, and the landing results were predicted to verify the feasibility of the device, providing a reference for the design of the lander and its buffer device.
\end{abstract}

\section{Introduction}

In modern times, aerospace powers have launched a series of detectors and landers [1-3] on the moon to explore the moon and tap its superior resources. [4-6] The stability of the lander is one of the key factors for its successful landing and subsequent detection missions. In addition, the landing probe carries multiple types of equipment, and its normal use requires a reasonable and effective landing buffer device to ensure a smooth landing of the lander. The research on lunar exploration in my country started late, and it is urgent to conduct indepth research on the lander buffer device.

The main innovation of this paper is for the lunar surface of a soft landing, and a discontinuous flexible ground modeling method is proposed, which completes the parameter matching of the lunar soil. Aiming at the aluminum honeycomb cushioning device of the lander, a discrete element modeling method is proposed. The mechanical properties and deformation mechanism of the discrete element honeycomb are analyzed and studied, and the feasibility of the discrete element modeling method is verified. On this basis, we propose a spider web honeycomb structure for a large lander, complete its modeling through a secondary development program, and test its discrete element structure.

Aluminum honeycomb is a new type of composite material developed in the middle of the last century. It plays an important role in buffering and absorbing energy in buffer lenders such as Apollo and Surveyor. For this reason, domestic and foreign scholars have launched a series of researches on its mechanical properties from the perspectives of theory, experiment, and numerical simulation. Gibson [7], Mc Farland [8], Ashby [9], and others explained the collapse behavior and 
deformation energy absorption mechanism of honeycombs in different directions and deduced the compressive strength, platform stress, folding wave, and structure parameters of honeycomb cells. The relationship between the parameters of the honeycomb material laid a theoretical foundation for the early honeycomb energy absorbers. Baker et al. [10] conducted static compression and dynamic impact experiments on thick-walled aluminum and stainless steel aluminum honeycombs. They found that the energy absorption of honeycombs is affected by the strain rate of the material, and the greater the strain rate, the greater the force on it. Hong [11] studied the effect of shear stress on the mechanical behavior of the honeycomb under static compression through a twoway loading experimental device. The experimental results show that the honeycomb strength under combined load is less than the honeycomb strength under pure compression load. They also analyzed the deformation mechanism of the honeycomb under combined loads and studied the relationship between the energy absorption rate and the sheer force. Zhang et al. [12] used the display nonlinear finite element software LS-DYNA to simulate the quasistatic compression process of the honeycomb and verified the accuracy of the finite element method. Levent et al. [13] developed three numerical methods based on the finite element method: honeycomb micromechanic simulation method, homogeneous material simulation method, and semiadaptive meshless smooth particle dispersion method. Jiangping Huang [14] from Harbin Institute of Technology has studied the relationship between the strength of aluminum honeycomb material and ambient temperature. Experimental data shows that the compressive strength of aluminum honeycomb material increases with the decrease of temperature. The reason for this result is aluminum foil and bonding. The strength of the agent is affected by temperature. Zhang [15] from Jiangsu University of Science and Technology used MSC-Dytran to study the crashworthiness of honeycomb panels, and the results showed that density has a greater impact on its performance. Zhang et al. [16] of Beijing Jiaotong University used the dynamic finite element software LS-DYNA to study the inplane impact characteristics of honeycombs with different cell structures and discussed their dynamic deformation processes. At the same time, they compared the effects of honeycomb panels under different impact speeds. The energy absorption characteristics and dynamic evolution process have concluded that the combined structure honeycomb has a stronger energy absorption effect under the same conditions. Quoc [17] studied the crushing behavior of the honeycomb structure from a theoretical perspective and then verified it by numerical simulation. Mertani [18] conducted an experimental study on the compression response of a hexagonal honeycomb core from an initial elastic state to a fully crushed state. The different specimens showed similar load/displacement curves, and the differences observed were only due to the influence of the core density, the number of cells, and specimens' size on the mechanical behavior of hexagonal honeycomb core which has a very important role in the energy absorption capacity. Xing [19] has manufactured a two-way corrugated aluminum honeycomb (BDCHA). First, five materials with different cell thickness/cell size ratios (t0/a) were designed, and compression tests were performed under quasistatic conditions. The results show that the initial peak stress and the plateau stress both increase with the increase of the t0/a ratio, and the plateau area determines the energy absorption performance. The research of aluminum honeycomb buffer is mainly divided into simulation research and experimental research. Most of the experiments focus on the triaxial compression experiment of aluminum honeycomb materials. At present, the mechanical properties of aluminum honeycombs with coplanar and different surfaces have been obtained through experiments; the simulation focuses on simulating its mechanical properties with finite element analysis software. And through LS-DYNA and other finite element dynamics calculation software, the impact dynamic simulation research was carried out, and the simulation calculation results were obtained. However, because of the rigid or semirigid assumption of the impact on the ground, the calculation result must ignore the energy absorption of the ground; so, the calculation result is only for reference.

To ensure the safe landing of the lander and protect the scientific load on it, a reasonable and effective landing buffer system and buffer are indispensable. According to the basic configuration, the landing buffer system is mainly divided into three categories: soft landing mechanism [20, 21], inflatable airbag buffer device, and aerial suspension. Different landing buffer systems have different characteristics and are adapted to different landing environments and mission requirements. In applications, the airbag cushioning method can carry a limited mass, and its airbag generally requires the deceleration effect of atmospheric resistance. During the contact with the landing surface, it is easy to be split and deflated by rocks, resulting in landing failure; the aerial suspension aircraft has a complex landing process and higher technical requirements, which is suitable for specific landing mission requirements, while the soft-landing mechanism is relatively simple, its stability is relatively reliable. It is the most widely used in lunar exploration landers. In the softlanding mechanism buffer system, the buffer is an important component for buffering and energy absorption. Currently, the buffers used in research and development mainly include aluminum honeycomb buffers, hydraulic buffers, mechanical buffers, magnetorheological fluid buffers, and metal rubber buffers. [22-28] The aluminum honeycomb buffer is the most used and most mature buffer among the successfully launched landers. The buffer has low mass, good energy absorption effect, stable operation, adjustable buffer stroke, and low dependence on the environment. The hydraulic shock absorber landed smoothly and reliably and has a long service life. It was widely used by surveyors and lunar series probes in the early lunar exploration climax. However, the problem of medium leakage was found in later tests, which limited its later application. The mechanical buffer is mainly based on the aircraft landing gear system, using springs, shock absorbers, and other elements to absorb impact energy. Mechanical buffer is a simple and reliable buffer that requires high precision. Magnetorheological fluid buffers have strong controllability, good repeatability, and good compatibility with current hydraulic systems. However, the technology is still in the development stage and has not yet been used. The metal rubber buffer is not sensitive to 
ambient temperature, but its performance stability is not good, and it has not been put into use so far.

Through the analysis of the current research progress and the existing problems in the research, the following conclusions are obtained: (1) aluminum honeycomb material is an anisotropic material with nonlinear large deformation. The current research is mainly based on finite element software for static or quasistatic simulation calculations, and there is little research on its dynamic impact. (2) In the study of aluminum honeycomb buffer, because of its ground rigidity or semirigid assumption on impact, the calculation result must ignore the energy absorption effect of the ground; so, the calculation result is only for reference.

The buffer device of the lander studied in this paper is a spider web buffer with an aluminum honeycomb as a filling material, which is suitable for the large lander. The outstanding results in this article are as follows: (1) establish a discrete element honeycomb bonding model, match its parameters through a quasistatic compression experiment, and compare the honeycomb mechanical characteristics and honeycomb deformation results in simulation and experiment under different compression directions. Verify the feasibility of the discrete element cellular simulation scheme. (2) Aiming at the large-scale lander, a spider web honeycomb buffer structure is proposed. According to the manufacturing process of the honeycomb, the discrete element modeling method is studied accordingly to obtain the particle position information and establish a spider web honeycomb model with a certain height. Finally, the overall compression simulation is used to verify the stability and simulation availability of the discrete element spider web honeycomb structure. (3) Establish the coupling model of the flexible lunar surface and the flexible lander with honeycomb buffer device, use EALINK to build the coupling simulation platform, realize the coupling calculation between the discrete element and multibody dynamics, and study the different working conditions during the landing process the landing buffer characteristics of the vehicle provide a reference for the structural design of the later lander.

\section{Materials and Methods}

\subsection{Discrete Element Method Modeling of the Aluminum} Honeycomb Structure. To study the mechanical properties and deformation of the aluminum honeycomb buffer structure during buffering, it is necessary to model and simulate the buffer material, use the discrete element method to model the honeycomb structure, refine the aluminum honeycomb material into particles, establish a discrete element honeycomb bonding model, and perform simulation analysis.

The modeling principle of the discrete element particle accumulation method is as follows. First, the honeycomb core material is divided and discretized (similar to the finite element mesh division), and then each unit after the division is simplified into spherical particles. Particles and particles are regarded as rigid small balls in soft contact, and there is extrusion in the direction perpendicular to the spherical surface, but the particles are rigid; so, there is an overlap between the particles in the calculation. The force between particles can

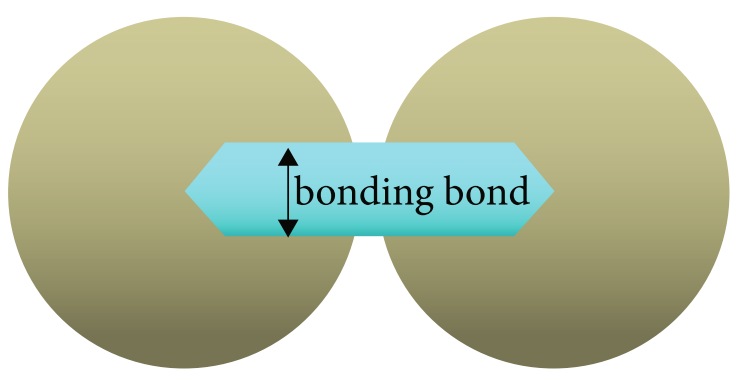

FIgURE 1: The basic unit of the whole aluminum honeycomb material is spherical particles, and the bonding bond connects the two spherical particles.

be calculated by the amount of overlap, elastic modulus, and material recovery coefficient. At the same time, the friction coefficient and the rolling friction coefficient are set in the tangential direction to control the friction between the particles, thereby affecting the fluidity of the material at yield. In addition, set up simulated bonding bonds between the particles. The formation of bonding bonds is the reverse process of the discretization of the core material. The bonding effect between the particles is used to ensure the accumulation of particles and form an organic whole. The force and motion state of the honeycomb panel can be distributed to each particle unit, as shown in Figure 1 below is the bonding bond model between the particles.

A columnar bonding bond is formed between the center positions of the particles to connect the particles. The bonding bond can record the force between the particles and reflect the transfer process of the force and the motion state. A columnar bonding bond is formed between the center positions of the particles to connect the particles. The bonding bond can record the force between the particles and reflect the transfer process of the force and motion state. The normal force on the bond, tangential force, normal moment, and tangential moment can be calculated according to the following: formulas (1)-(4)

$$
\begin{gathered}
\delta F_{n}=-v_{n} S_{n} A \delta t, \\
\delta F_{t}=-v_{t} S_{t} A \delta t, \\
\delta M_{n}=-\omega_{n} S_{t} J \delta t, \\
\delta M_{t}=-\omega_{t} S_{n} \frac{J}{2} \delta t,
\end{gathered}
$$

where

$A$ is the cross-sectional area of the bonding bond, $\left(\mathrm{m}^{2}\right)$.

$J$ is the moment of inertia of the cross section of the bond concerning the center of the circle, $J=1 / 2 \pi R_{B}{ }^{4}\left(\mathrm{~m}^{4}\right)$.

$R_{B}$ is the radius of the bonding bond (m).

$\delta t$ is the time step (s).

$S_{n}$ and $S_{t}$ is the normal stiffness and shear stiffness $\left(\mathrm{N} / \mathrm{m}^{3}\right)$.

$v_{n}$ and $v_{t}$ is the normal and tangential velocity of the particles $(\mathrm{m} / \mathrm{s})$.

$\omega_{n}$ and $\omega_{t}$ is the normal and tangential angular velocity $(\mathrm{rad} / \mathrm{s})$ 


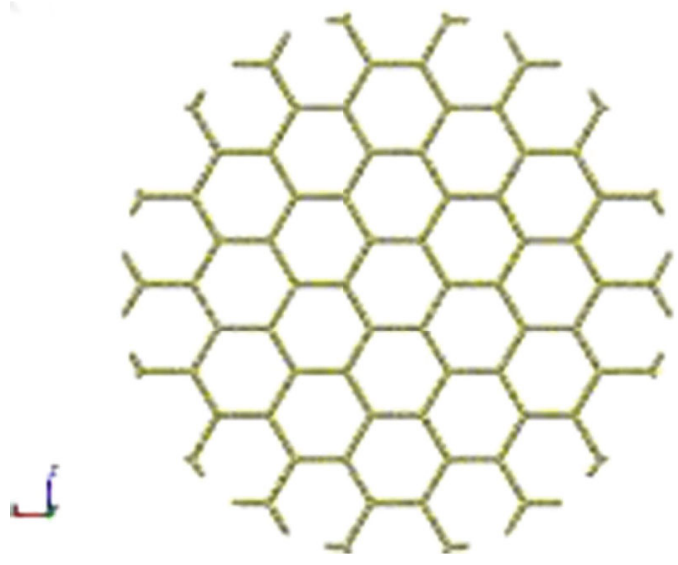

(a)

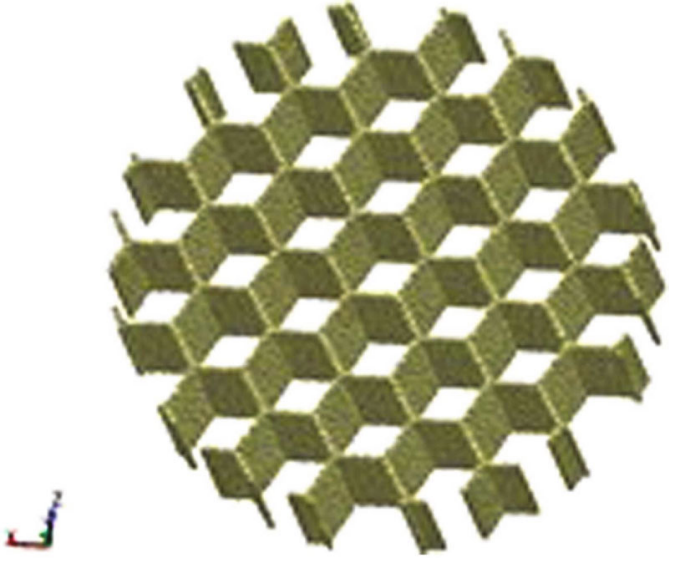

(b)

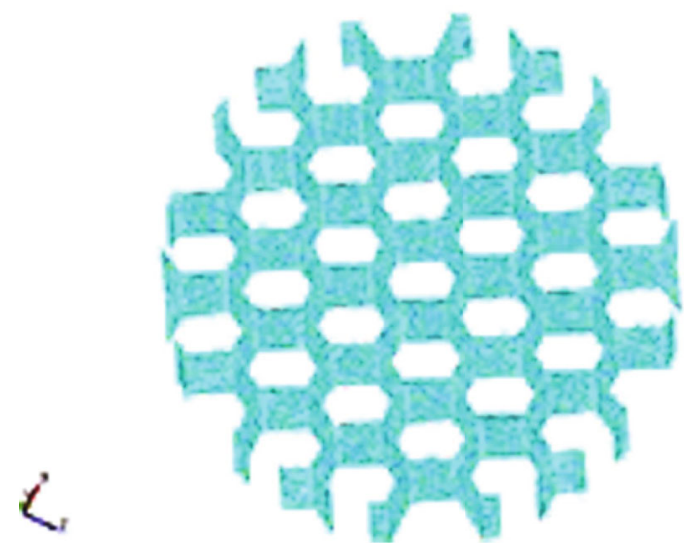

(c)

Figure 2: Discrete element model of aluminum honeycomb and its bonding structure. (a) Discrete element model of ideal Aluminum honeycomb grid. (b) Discrete element model of bent aluminum foil. (c) Discrete element model of Aluminum honeycomb structure.

When the force of the bonding bond is too large and exceeds the maximum stress, see the following: equations (5) and (6). The bonding bond is broken, and the particles are no longer affected by the bonding force. The force of its movement is following the Hertz Medellin Model calculation.

$$
\begin{gathered}
\sigma_{\max }<\frac{-F_{n}}{A}+\frac{2 M_{t}}{J} R_{B}, \\
\tau_{\max }<\frac{-F_{t}}{A}+\frac{M_{n}}{J} R_{B} .
\end{gathered}
$$

In EDEM, a suitable model is established according to the parameter ratio of the aluminum honeycomb panel, and the honeycomb particles are connected to form through the bonding bonds between the particles, and finally, the discrete element model of the aluminum honeycomb and its bonding structure is shown in Figure 2 below.

\subsection{Research on the Structure of Cobweb Honeycomb Cushioning Device}

2.2.1. Cobweb Honeycomb Buffer Device Structure. The spider web in nature can effectively transmit it to the entire web surface when subjected to impact force, and it is not

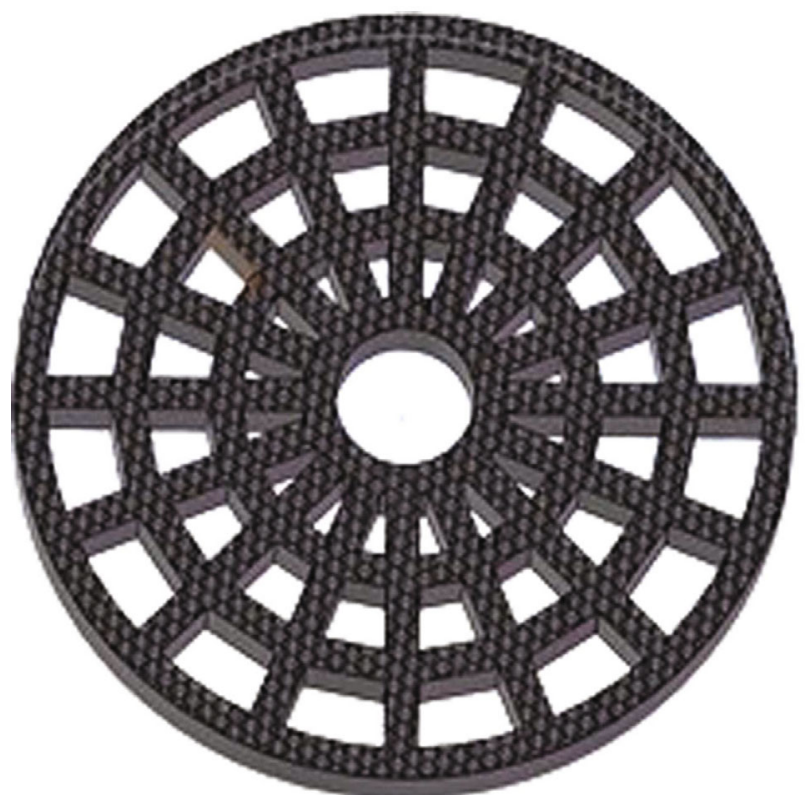

Figure 3: The structure of the spider web buffer device. 


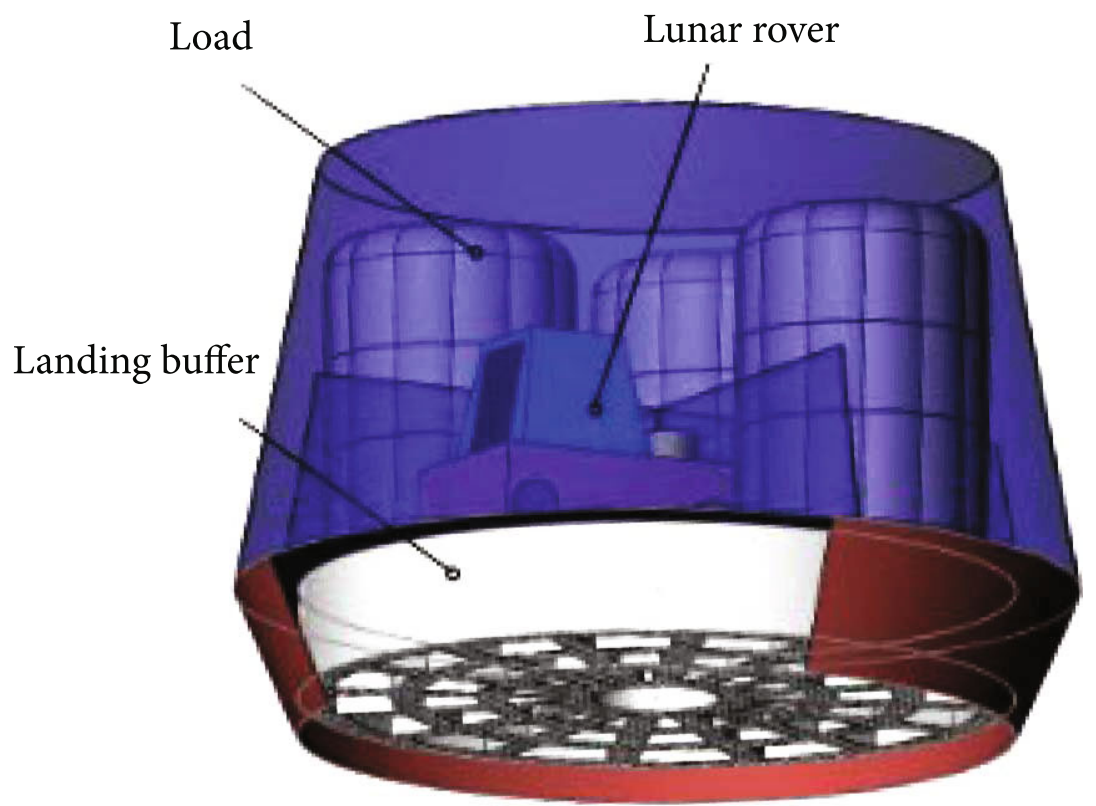

Figure 4: The schematic diagram of the whole lander.

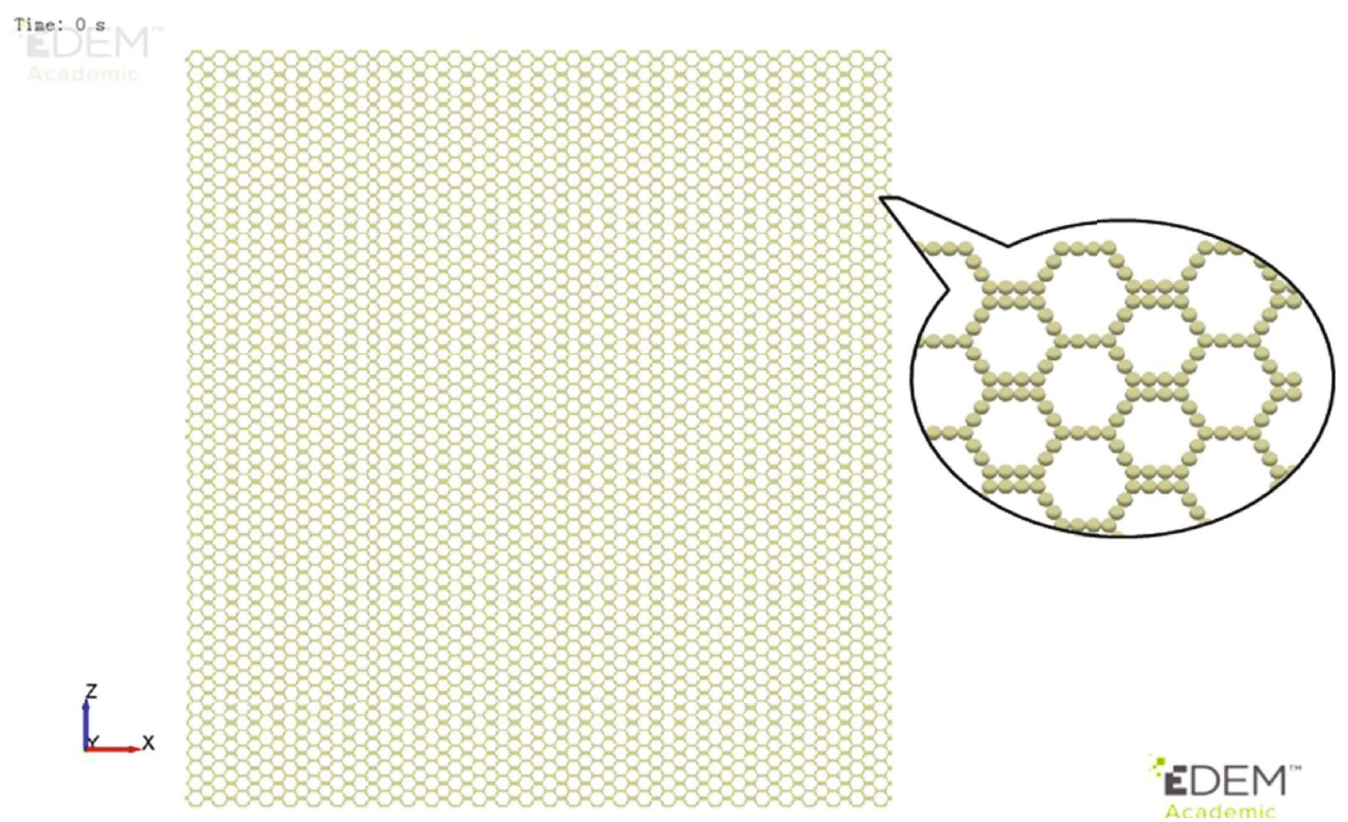

Figure 5: Discrete element model of single-layer honeycomb panel.

easy to cause local damage when dissipating the impact energy. It is a typical stable tension structure. Because of this characteristic, the structure of the spider web honeycomb cushioning device is designed as shown in Figure 3. Its overall configuration is similar to that of the spider web. The inside of the structure is composed of evenly distributed hexagonal honeycombs, which are applied to the bottom of the lander, and achieves buffering and energy absorption. Figure 4 shows a schematic diagram of the entire lander. It carries a lunar rover and various loads. The landing mass is large, and the impact kinetic energy during descent is large. However, the cobweb honeycomb buffer device has a large contact surface with the lunar surface; so, it passes through the honeycomb. The landing kinetic energy absorbed by the deformation is also larger, and finally, a soft landing is realized.

2.2.2. Discrete Element Modeling of Cobweb Honeycomb Buffer Device. Considering the particularity of discrete element modeling, the spider web honeycomb buffer structure 

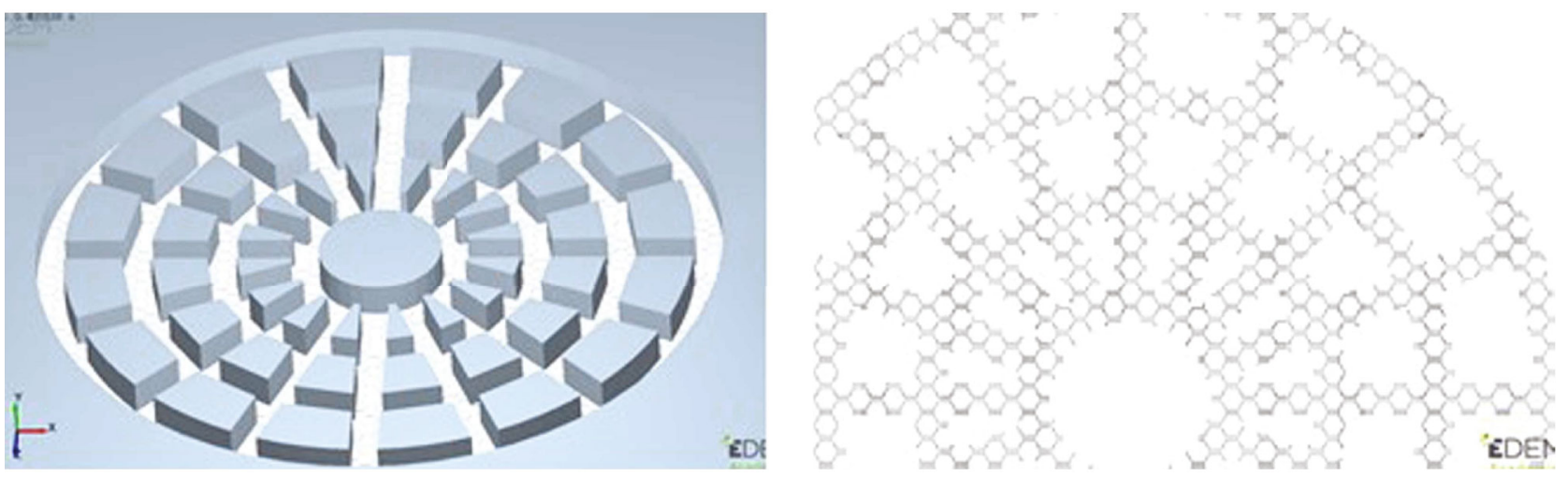

Figure 6: Honeycomb stamping die and its discrete element model after stamping.

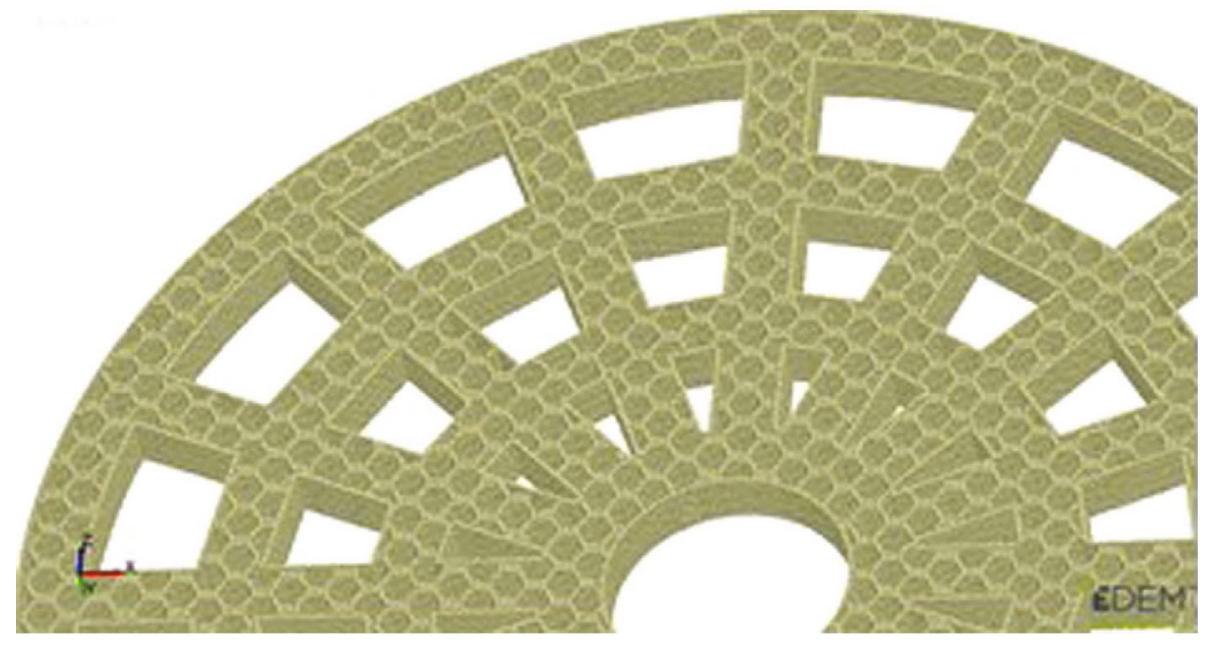

Figure 7: Discrete element model of three-dimensional spider web honeycomb.

used in its simulation is composed of a large number of honeycomb particles. The traditional discrete element modeling method is relatively cumbersome.

Therefore, use EDEM secondary development to model it. Array the position points of a single honeycomb in the vertical and horizontal directions to obtain the position coordinates of all the particles on the honeycomb plate, establish the corresponding particle model in EDEM, use the secondary development program to generate honeycomb particles at the specified position, and finally, get the discrete element model of the cut single-layer honeycomb panel that is shown in Figure 5 below.

To obtain the spiderweb-like honeycomb structure, a stamping die with a complementary shape is established, which is introduced into the EDEM, and the die is used to impact the single-layer honeycomb panel to remove the excess part of the square honeycomb and leave the particle information for future use. The honeycomb stamping die and the discrete element model after stamping are shown in Figure 6.

The position information of the lamellar particles of the spider web honeycomb buffer structure can be obtained from the above model. Export it as a configuration file, use the secondary development of EDEM to superimpose and accumulate the particle flakes in the height direction, and bond the entire three-dimensional cobweb honeycomb structure. The obtained spider web-like buffer honeycomb structure is shown in Figure 7 below.

2.3. The Whole Lander Simulation Model. The coupling model needs to be established before the cosimulation. First, establish the discrete element lunar soil model [29-31] in EDEM. The lunar soil is mainly composed of a large number of granular materials. The particle size distribution is mainly below $1 \mathrm{~mm}$, and the particle shape is complex and changeable. From regular spherical to irregular polygonal shapes, such as elongated, concave-angled, and subangular, these complex lunar soil shapes can be combined with spherical particles of different sizes and positions in discrete element EDEM, as shown in Figure 8. These complex shapes are superimposed by several spherical particles. For lunar soil particles with more surface edges and more complex shapes, the greater the number of subparticles that make up them. The lunar soil formed by these complex shapes can simulate the mosaic effect between the lunar soils and make the model properties different from each other. The real lunar 

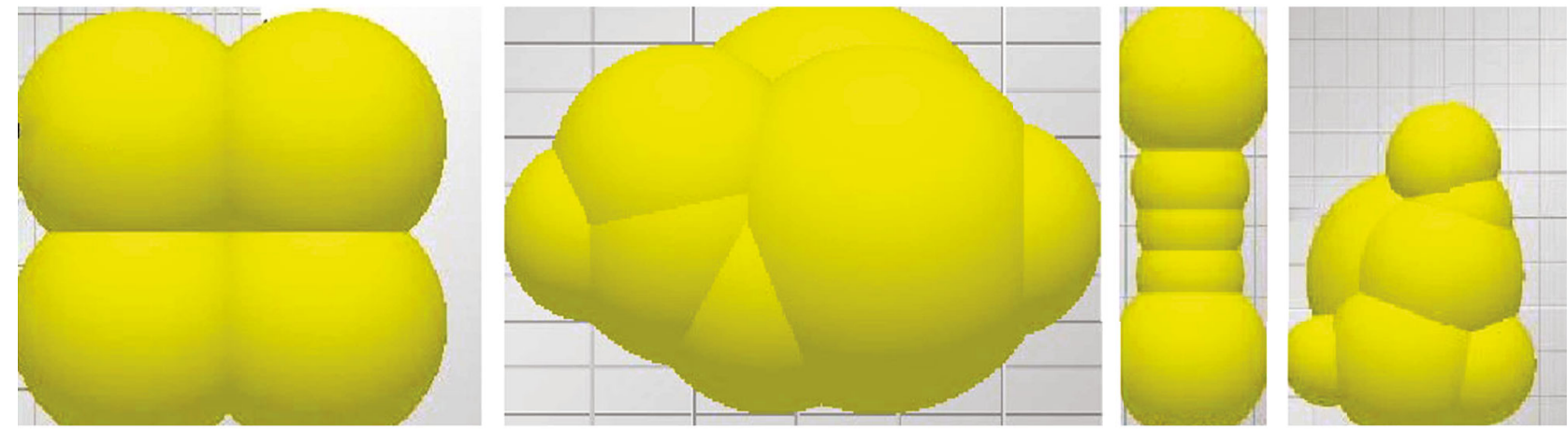

Figure 8: The simulated lunar soil particles established in EDEM.

soil is highly consistent, but it also greatly increases the number of particles, and more and more complex surface contacts are formed between the subparticles, which will inevitably increase the calculation difficulty of the simulation in the later parameter matching and mechanical testing, extending the calculation time. Therefore, it is necessary to replace the particle cluster model with complex shapes and replace the irregular particle model with the spherical particle model for calculation. By adjusting the friction coefficient between the spherical particles, the embedding force and surface friction between the irregularly shaped particles are equivalent. Force and cohesion finally make spherical particles and irregular particles have the same mechanical properties. A single spherical discrete element lunar soil model as shown in Figure 9 is established using simple spherical particles. The model is cylindrical as a whole and is composed of a total of 100,000 single spherical particle models. The lunar soil particles have the same size and surface contact. In spherical contact, the middle protrusion part simulates the highland plain of the lunar surface. The particle composition of this model is single, which can better show the isotropy of the lunar soil.

The single spherical discrete element lunar soil model has greater advantages in simulating a smaller area of lunar soil. The particle size and structure are single, and it is easier to achieve in the later parameter mechanical performance calibration. However, in discrete element simulation, when the lunar soil covers a large area and the accuracy of the simulation results is required, millions of lunar soil particles need to be generated. Although increasing the diameter of lunar soil spherical particles can reduce the number of particles, correspondingly, as the diameter of the particles increases, the radius of the spherical surface in contact with the lender also increases, pores increase, and the actual pressure-bearing area decreases. The lunar soil model cannot fully contact the lander, and the simulation result has a large error. To make the simulation results more realistic and reliable, and at the same time to reduce the amount of calculation, this paper adopts the axial and radial stepwise magnification method to model the lunar soil. At the position where the impact is directly in contact, a smaller radius is formed. Lunar soil particles gradually enlarge the size of lunar soil particles in the axial and radial positions away from the impact point. For lunar soil spherical particles with

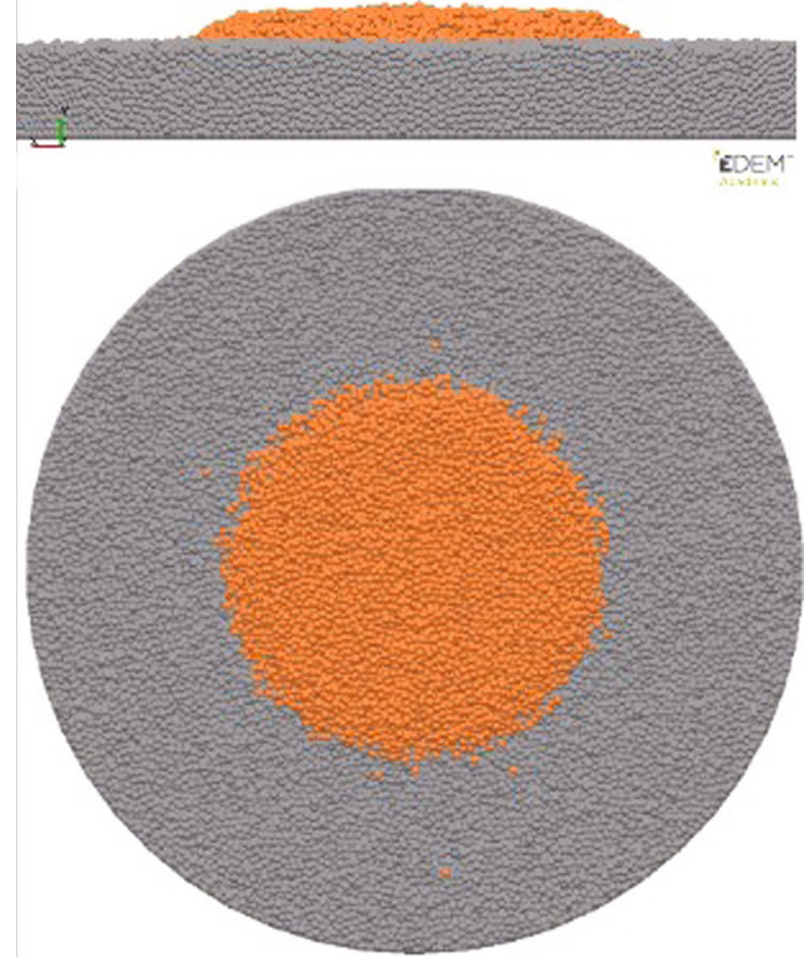

FIGURE 9: Single spherical discrete element lunar soil model.

different sizes and radii, the thickness of the lunar soil also shows a progressive relationship, achieving a full reduction in the number of model particles and increasing the purpose of calculating speed. In addition, the final shape and size of the lunar soil also have a certain impact on the accuracy and speed of the simulation. Because the impact is evenly radiated from the impact point to the surroundings, the overall shape of the lunar soil is set to a cylindrical shape, making the impact process during landing in the middle, and the force received by the lunar soil is evenly transmitted to the surrounding area, reducing the boundary effect caused by uneven diffusion. On the premise of effectively eliminating the boundary effect, to further reduce the amount of calculation, the diameter of the cylinder is set to 5 times the largest radial dimension of the lander body. 


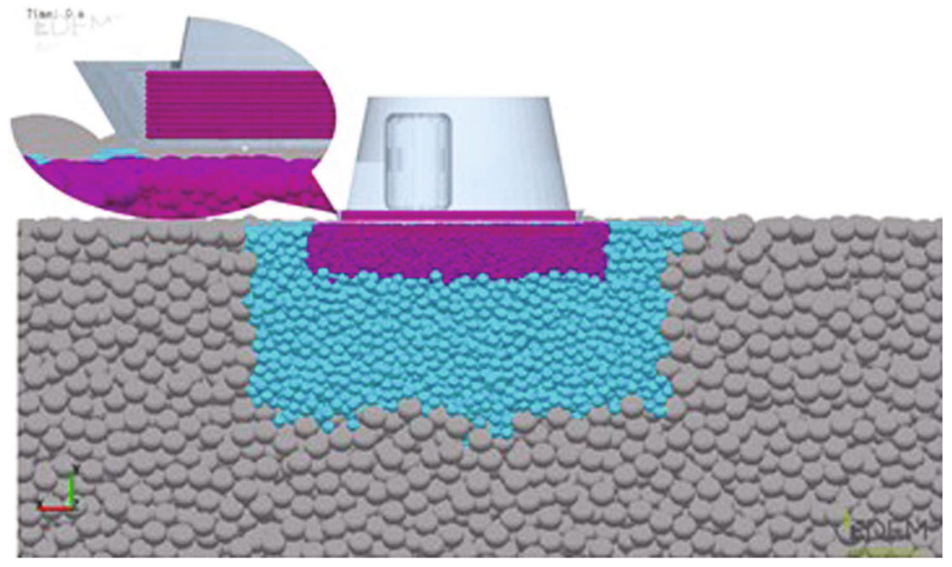

FIgURE 10: Schematic diagram of EDEM lander-honeycomb-lunar soil coupling model.

TABle 1: Discrete element parameters of cobweb honeycomb.

Models
Bonding bond model
Discrete element model of aluminum
honeycomb and its bonding structure
Discrete element model of three-
dimensional spider web honeycomb

A columnar bonding bond is formed between the center positions of the particles to connect the particles. The bonding bond can record the force between the particles and reflect the transfer process of the force and motion state.

A suitable model is established according to the parameter ratio of the aluminum honeycomb panel, and the honeycomb particles are connected to form aluminum honeycomb panel, through the bonding bonds between the particles

Array the position points of a single honeycomb in the vertical and horizontal directions to obtain the position coordinates of all the particles on the honeycomb board, establish the corresponding particle model in EDEM, and obtain the discrete element model of the uncut single-layer honeycomb board. Establish a stamping die complementary to its shape, import it into the EDEM, and use the die to impact the single-layer honeycomb panel to obtain a spiderweb-like cushion honeycomb structure.

Single spherical grain lunar soil model

EDEM lander-honeycomb-lunar soil coupling model
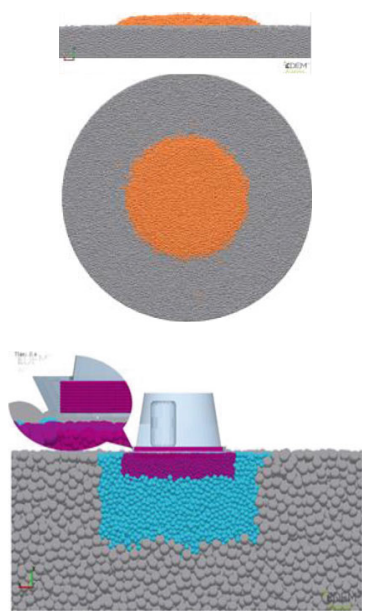

The lunar soil is modeled by an axial and radial magnification step by step, and the lunar soil particles with a smaller radius are formed at the positions directly contacted during the impact. In the axial and radial directions away from the impact point, the particle size of the lunar soil is gradually enlarged. In lunar soil spherical particles of different sizes and radii, the thickness of the lunar soil also shows a progressive relationship, achieving a full reduction in the number of model particles

Based on the established lunar soil model, the lander prototype was imported into the lunar soil discrete element model according to certain reference system rules. 


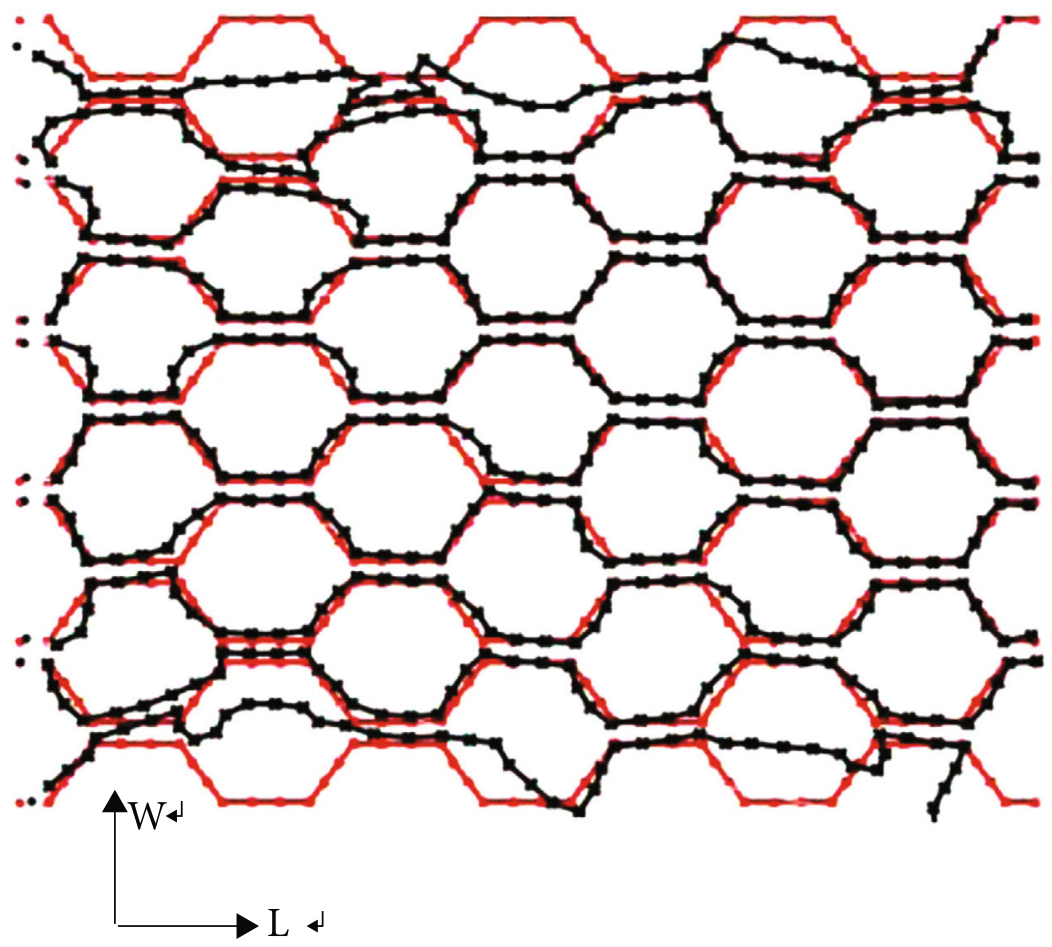

$\longrightarrow$ Before deformation

$\longrightarrow$ After deformation

Figure 11: The structure of the square honeycomb before and after the deformation of the upper surface when the different faces are compressed.

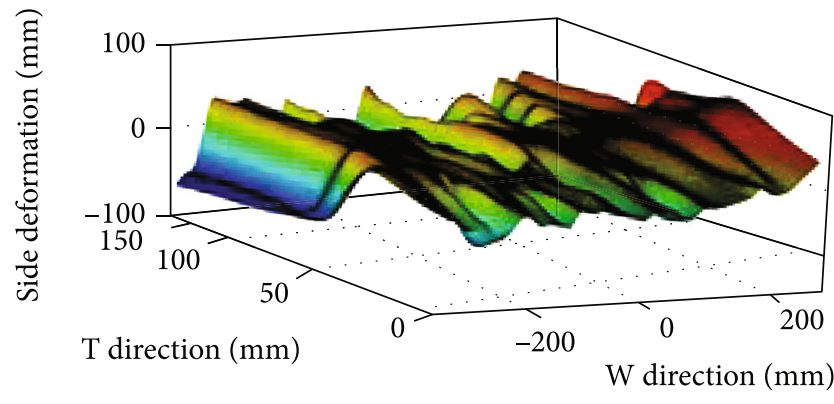

(a) $W$ surface deformation

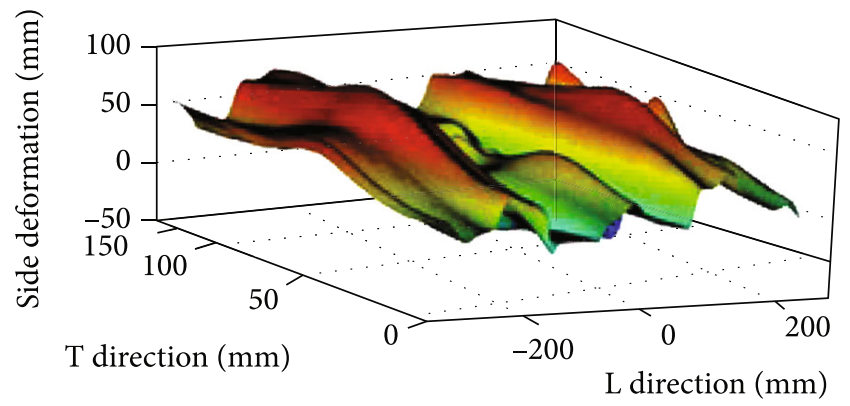

(b) $L$ surface deformation

FIgURE $12: W$ and $L$ surface deformation fitting diagram.

Adjust the recovery coefficient, static friction coefficient, and rolling friction coefficient of the lunar soil in the discrete element according to the lunar soil accumulation angle obtained by the relevant scholars $[32,33]$ in the past. In the end, this paper selected the accumulation angle of $37^{\circ}$, the static friction coefficient of 0.44 , the rolling friction coefficient of 0.2 , and the recovery coefficient of 0.7 as the model parameters of the lunar soil model. Based on the established lunar soil model, the lander prototype was imported into the lunar soil discrete element model according to certain reference system rules. Finally, with the help of the secondary development program in EDEM, honeycomb particles are generated at the particle position of the spider web honey- comb. The height of the honeycomb is exactly the height of the filling area inside the buffer device in the lander. Finally, the honeycomb particles are bonded and parameters are adjusted. The lander-honeycomb-lunar soil coupling model in EDEM is shown in Figure 10 below.

The models, modeling methods, and method introductions mentioned above are shown in Table 1.

\section{Results and Discussions}

3.1. Discrete Element Simulation of the Aluminum Honeycomb Structure. The discrete element honeycomb obtained by matching with the experiment is consistent with the actual 


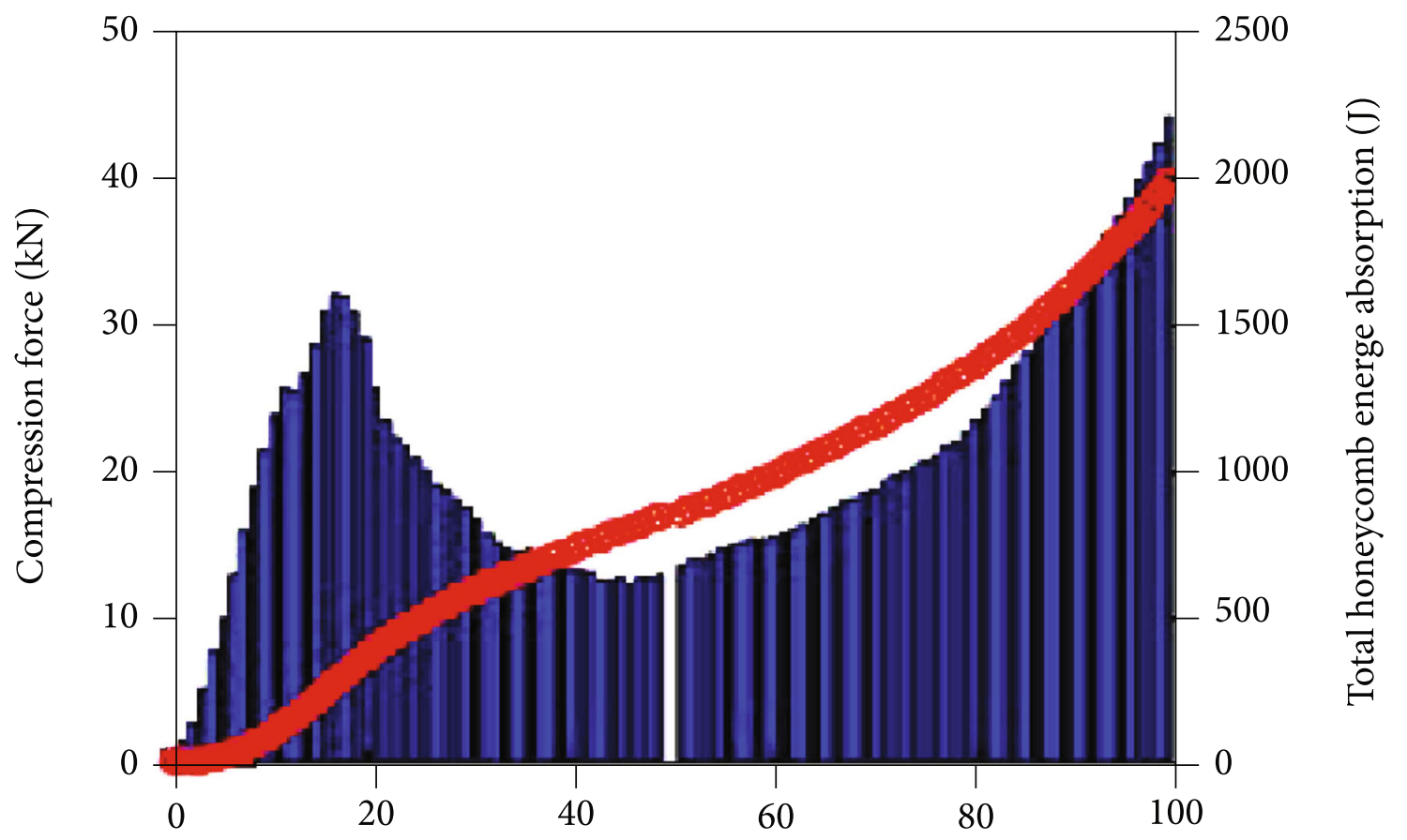

Compression displacement $(\mathrm{mm})$

\section{- Total honeycomb energy absorption \\ Compression force}

FIGURE 13: Honeycomb energy absorption curve when compressed on different surfaces.
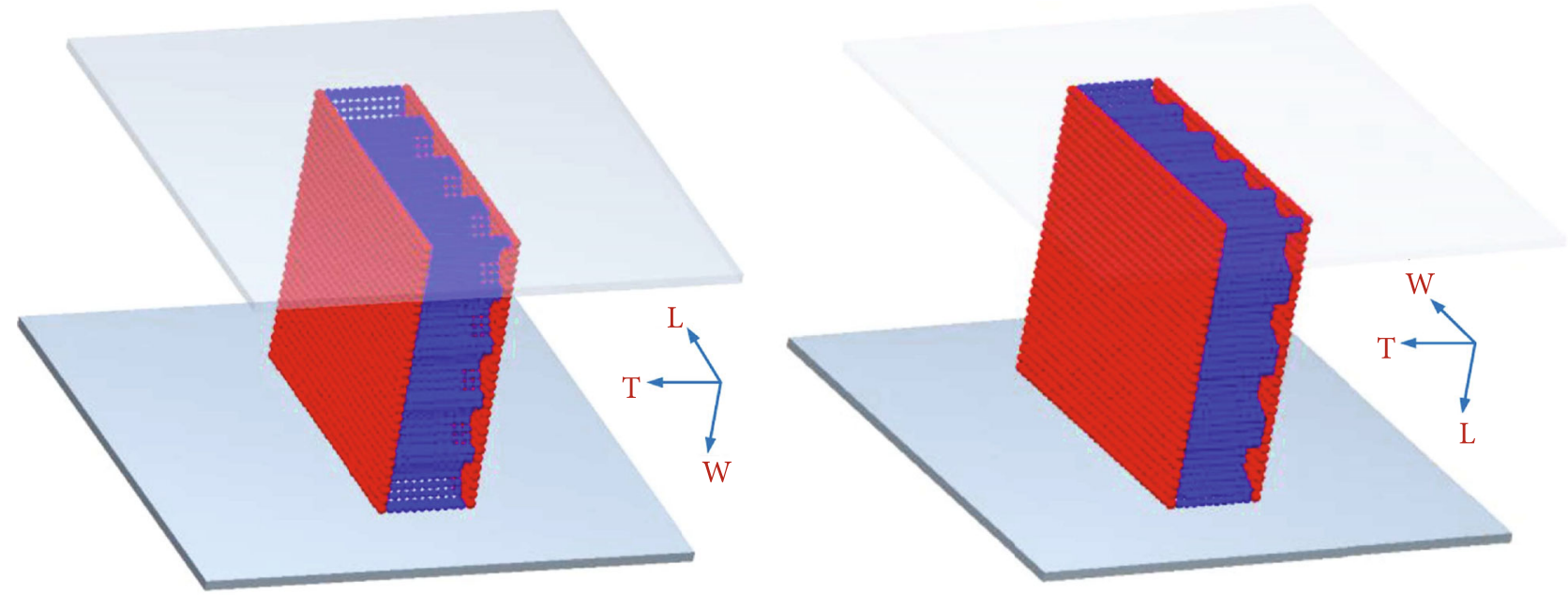

Figure 14: Discrete element square honeycomb coplanar compression model.

honeycomb in terms of mechanical properties. In this section, the simulation deformation of the discrete element honeycomb will be studied, and the deformation results of out-of-plane compression and coplanar compression will be analyzed.

\subsubsection{Different Surface Compression Deformations of Discrete} Element Honeycomb. A uniform load was applied to the upper surface of the square honeycomb, and axially different plane compressions were performed, the particle positions before and after the deformation of the upper surface were extracted for comparison, and the deformation results of the structure were obtained as shown in Figure 11 below.

From the results before and after the honeycomb structure is deformed, it can be seen that the deformation of the 


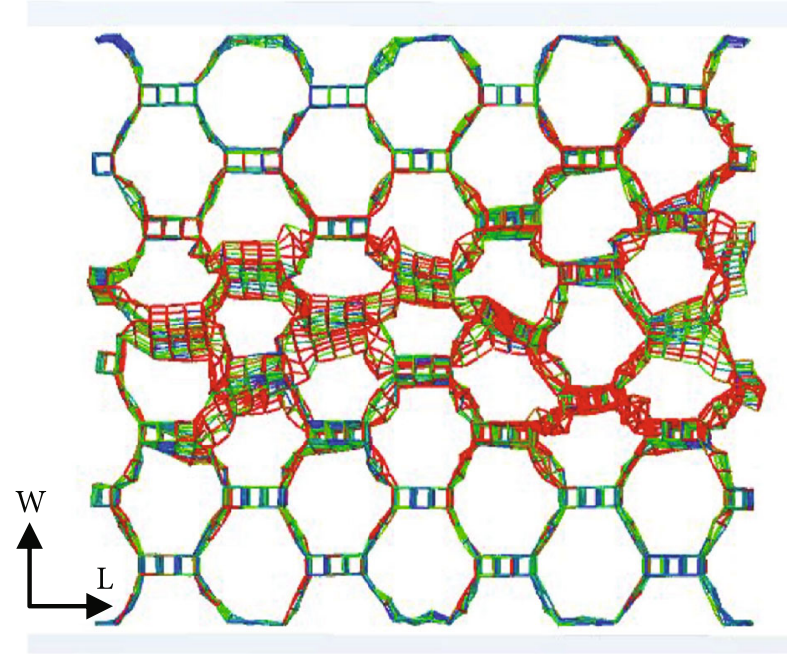

(a)

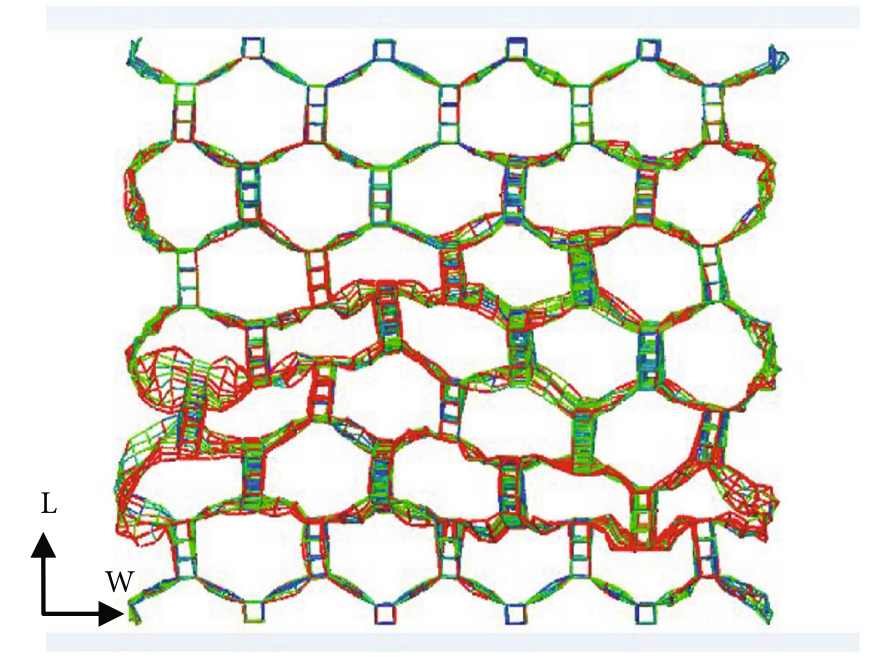

(b)

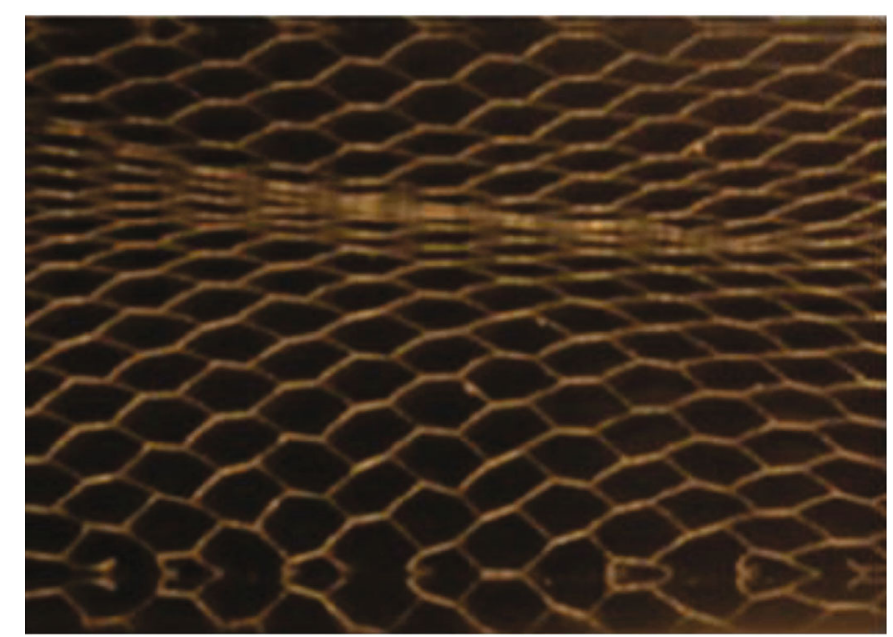

(c)

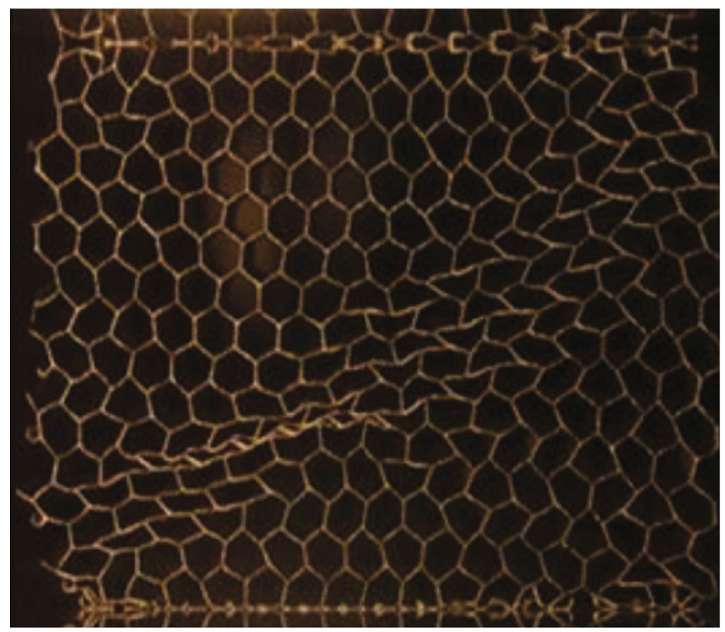

(d)

FIGURE 15: Simulation and experimental comparison of coplanar compression deformation of a square honeycomb. (a) $W$ direction deformation of simulated honeycomb. (b) $L$ direction deformation of simulated honeycomb. (c) Deformation in the $W$ direction of the experimental honeycomb. (d) Deformation in the $L$ direction of the experimental honeycomb.

hexagonal structure at the edge of the honeycomb is greater than the inside of the honeycomb core, and the deformation in the $W$ and $L$ directions are also different. Therefore, at the same time, extract a layer of honeycomb core side particles in the $W$ and $L$ directions. According to the positions of the particles before and after the deformation, calculate the deformation at each position on the surface and use MATLAB to perform surface fitting on the deformation at different positions to obtain the deformation results of the square honeycomb $W$ surface and $L$ surface that are shown in Figure 12 below.

In the EDEM postprocessing, the compression force received by the honeycomb during the compression process is extracted, the compression displacement is evenly divided, the honeycomb energy absorption in the local compression displacement is calculated according to the relationship between displacement, force, and energy, and the area is summed. The energy absorption curve of the honeycomb during the entire compression process is shown in Figure 13 below. This is in response to Zhang [34] research.

In the early stage of compression, the compression force increases linearly, and the honeycomb energy absorption increases rapidly. After entering the honeycomb collapse zone, the compression force remains stable, and its energy absorption increases linearly to achieve stable energy absorption. After entering the compaction zone, its compression force rises rapidly. The total amount of energy absorption has also increased dramatically.

\subsubsection{Coplanar Compression Deformation of Discrete} Element Honeycomb. Aluminum honeycomb is an anisotropic material, and its coplanar and different face mechanical properties and deformation are quite different. For this reason, the $W$ direction and $L$ direction of the square honeycomb were tested for coplanar compression. Figure 14 below shows the discrete element schematic diagram of the 


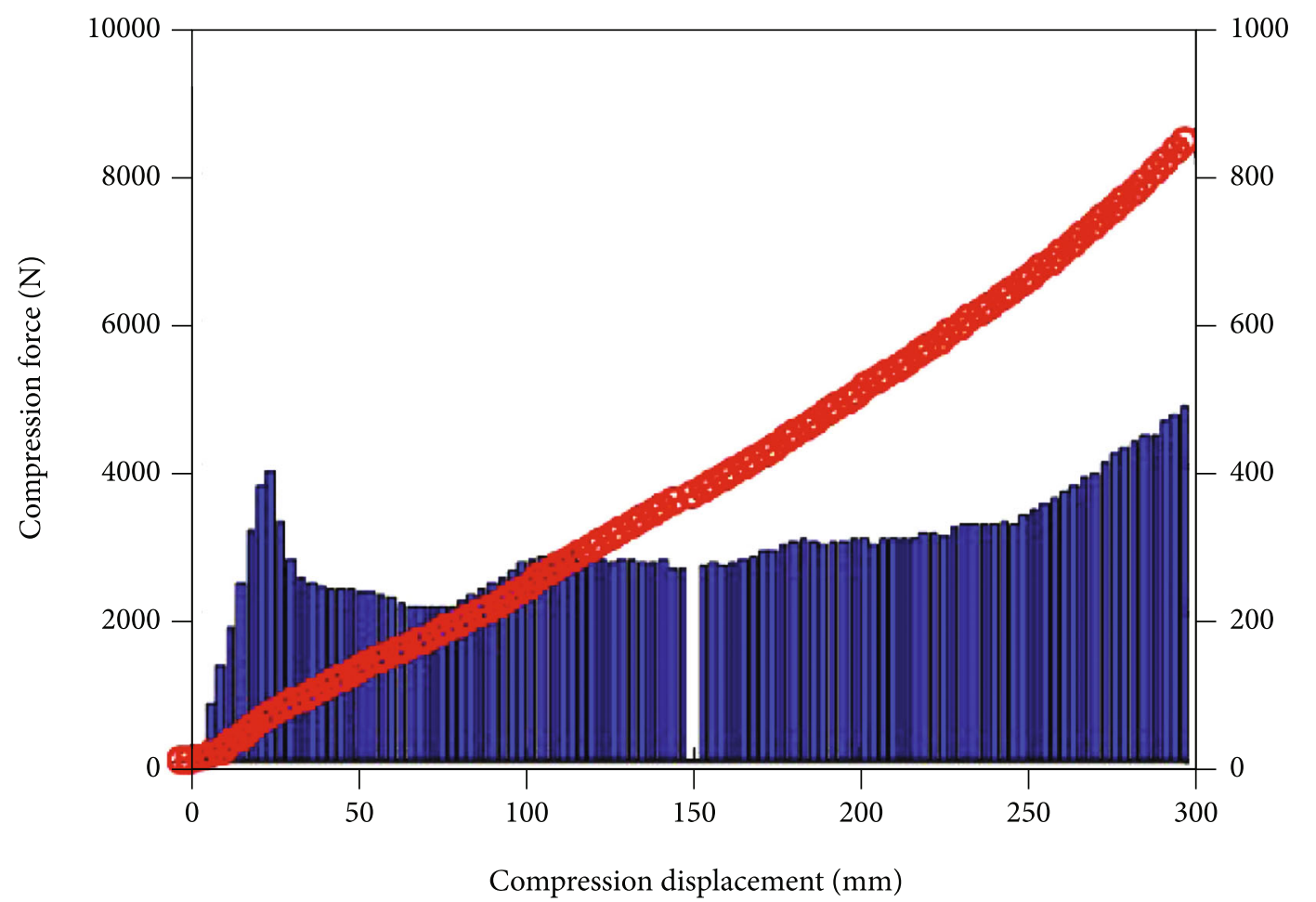

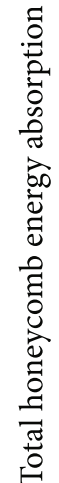

(a) $W$ direction

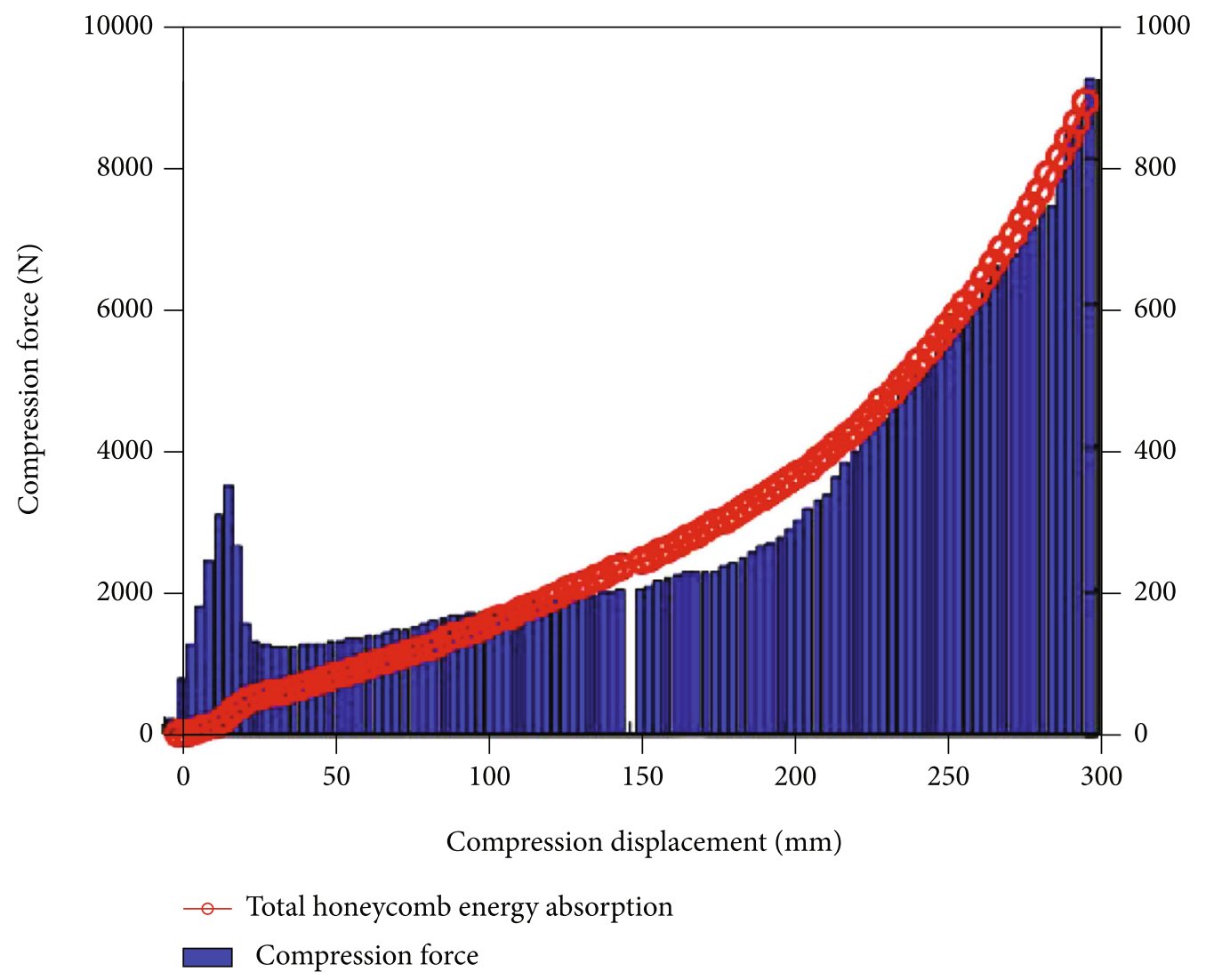

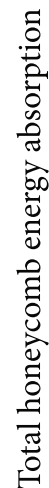

(b) $L$ direction

FIGURE 16: Honeycomb energy absorption curve under coplanar compression. 
TABLe 2: Discrete element parameters of cobweb honeycomb.

\begin{tabular}{|c|c|c|c|c|c|}
\hline Cell height (mm) & Volume stiffness $\left(\mathrm{N} / \mathrm{m}^{3}\right)$ & Shear stiffness $\left(\mathrm{N} / \mathrm{m}^{3}\right)$ & Bonding radius $(\mathrm{mm})$ & Compression speed $(\mathrm{mm} / \mathrm{s})$ & Time step (s) \\
\hline 160 & $10^{10}$ & $5 \times 10^{9}$ & 2 & 100 & $5 \times 10^{-6}$ \\
\hline
\end{tabular}

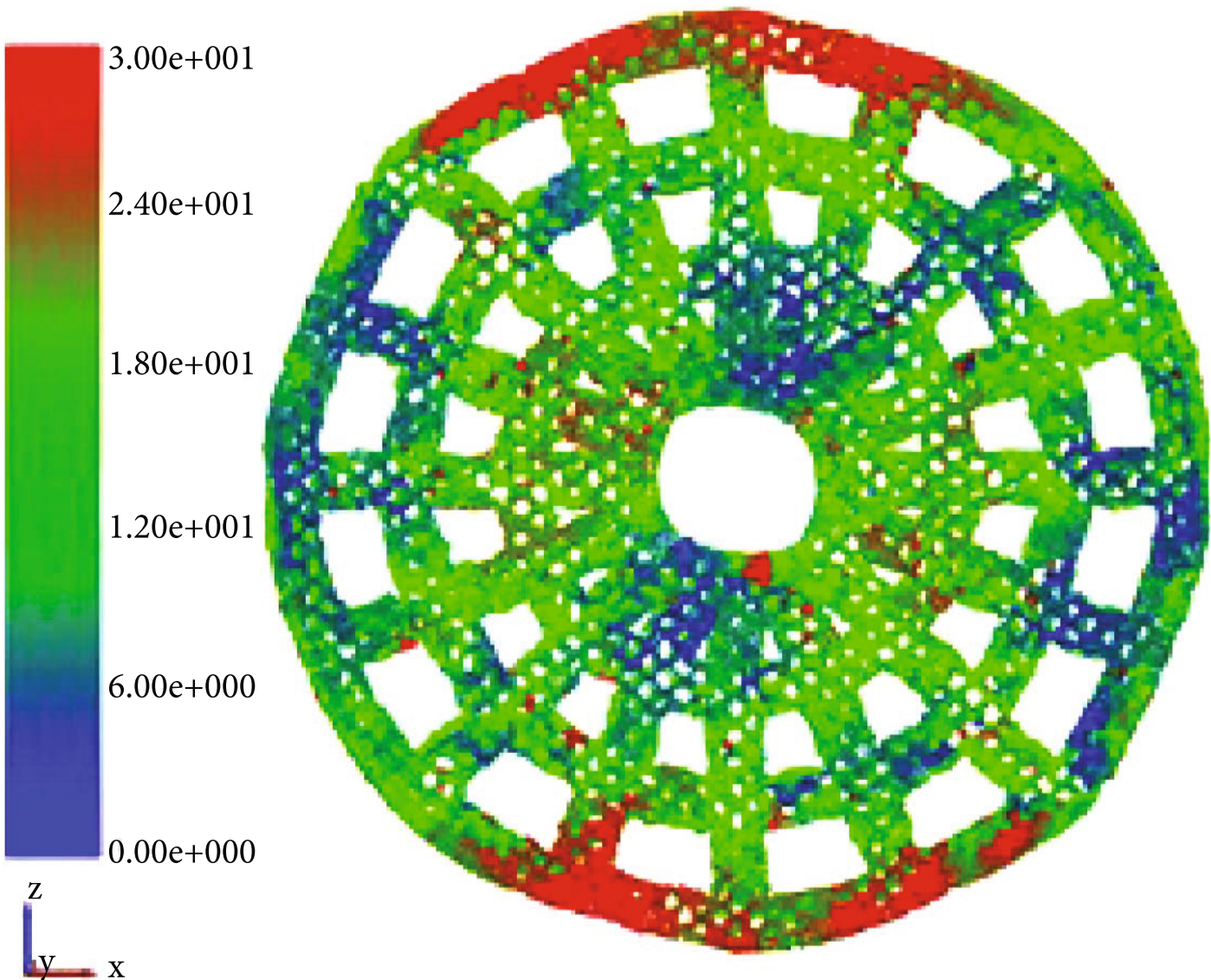

FiguRE 17: Compression deformation of spider web honeycomb.

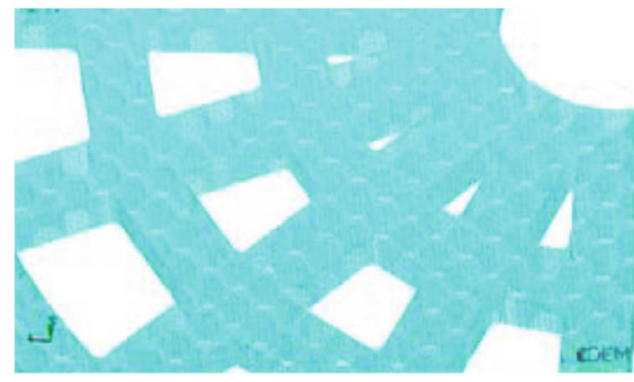

(a)

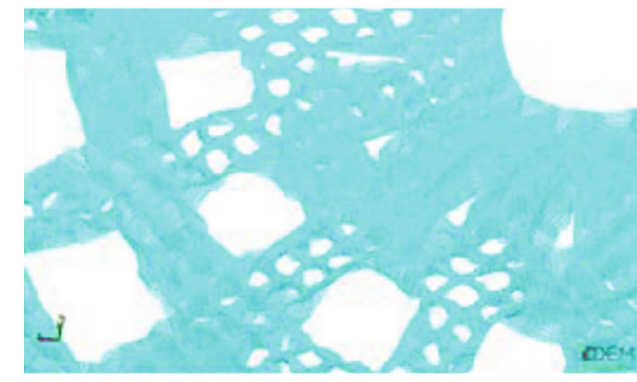

(b)

Figure 18: Deformation of bonding structure before and after compression of spider web honeycomb. (a) Cobweb honeycomb structure before compression. (b) Cobweb honeycomb structure after compression.

coplanar compression model of a square honeycomb in the $W$ and $L$ directions.

In EDEM, the pressing plate is set to move in a uniform linear motion to apply a uniform load to the two sides of the square honeycomb, and the deformation results of the simulated honeycomb core in the $W$ and $L$ directions are shown in Figures 15(a) and 15(b) below. Ines et al. [35] conducted a coplanar compression experiment on the aluminum honeycomb and obtained the deformation in the $W$ direction and the $L$ direction as shown in Figures 15(c) and 15(d) below. The results of this experiment correspond to Tanaka's [36] research. 


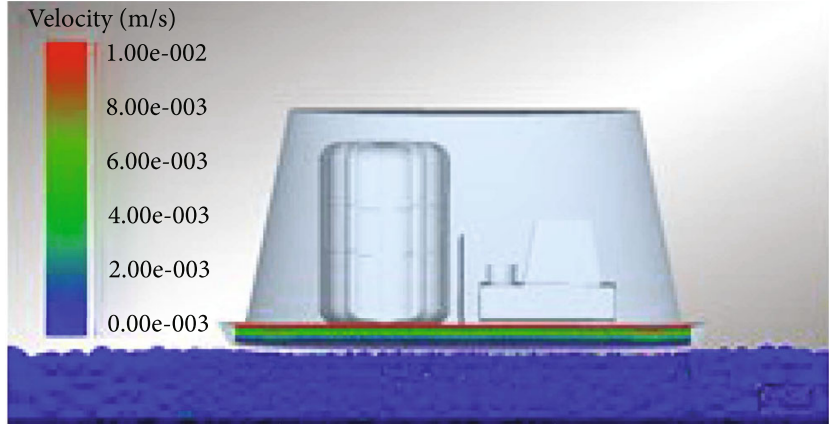

(a) $t=0 \mathrm{~s}$

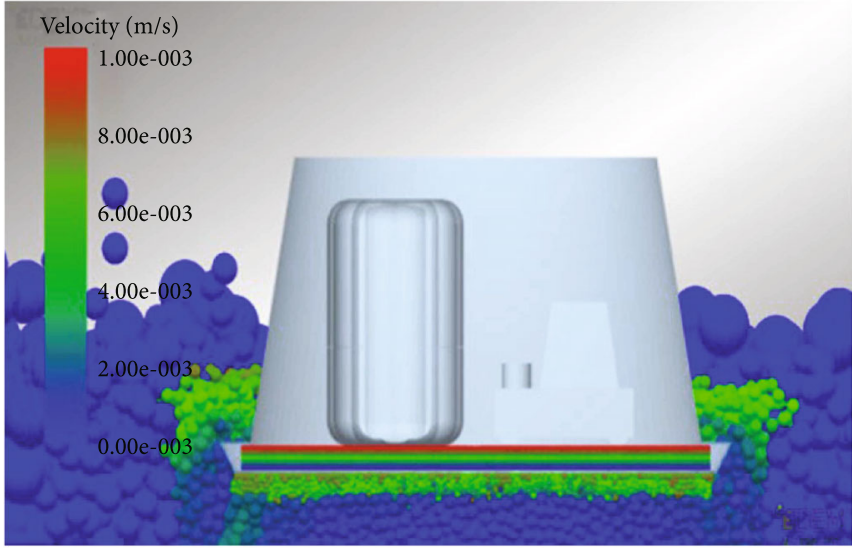

(c) $t=0.1 \mathrm{~s}$

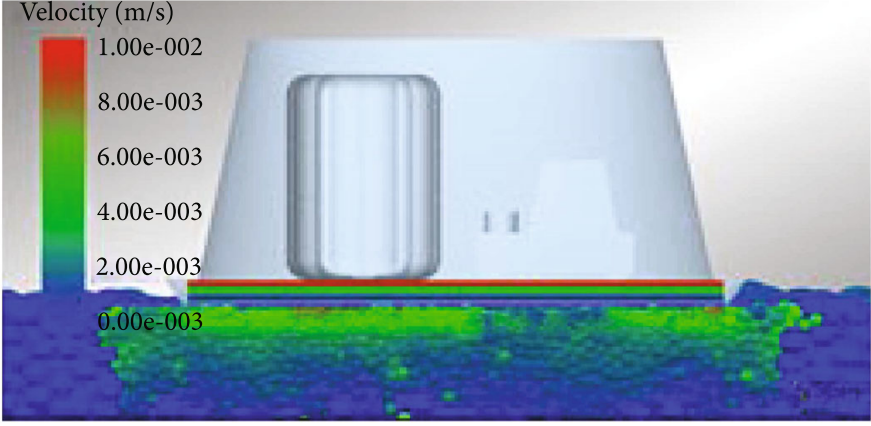

(b) $t=0.05 \mathrm{~s}$

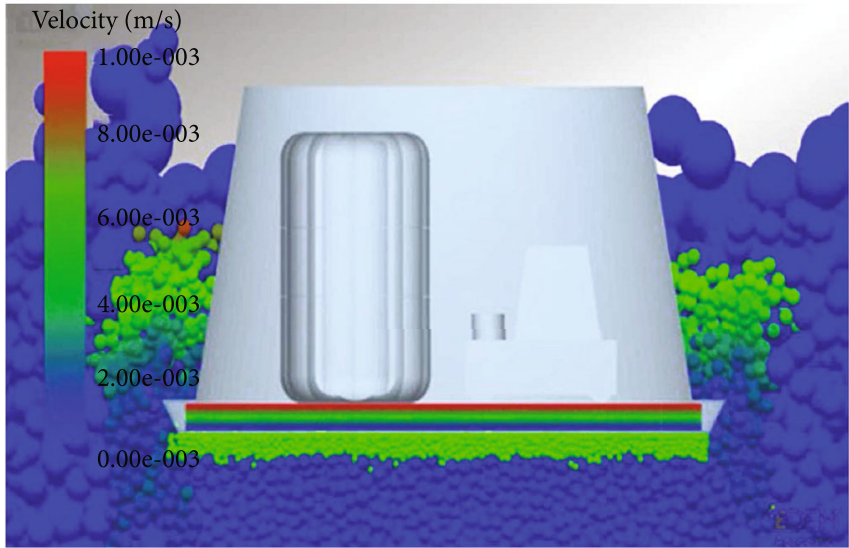

(d) $t=0.15 \mathrm{~s}$

FIgURE 19: The landing simulation process of the whole lander.

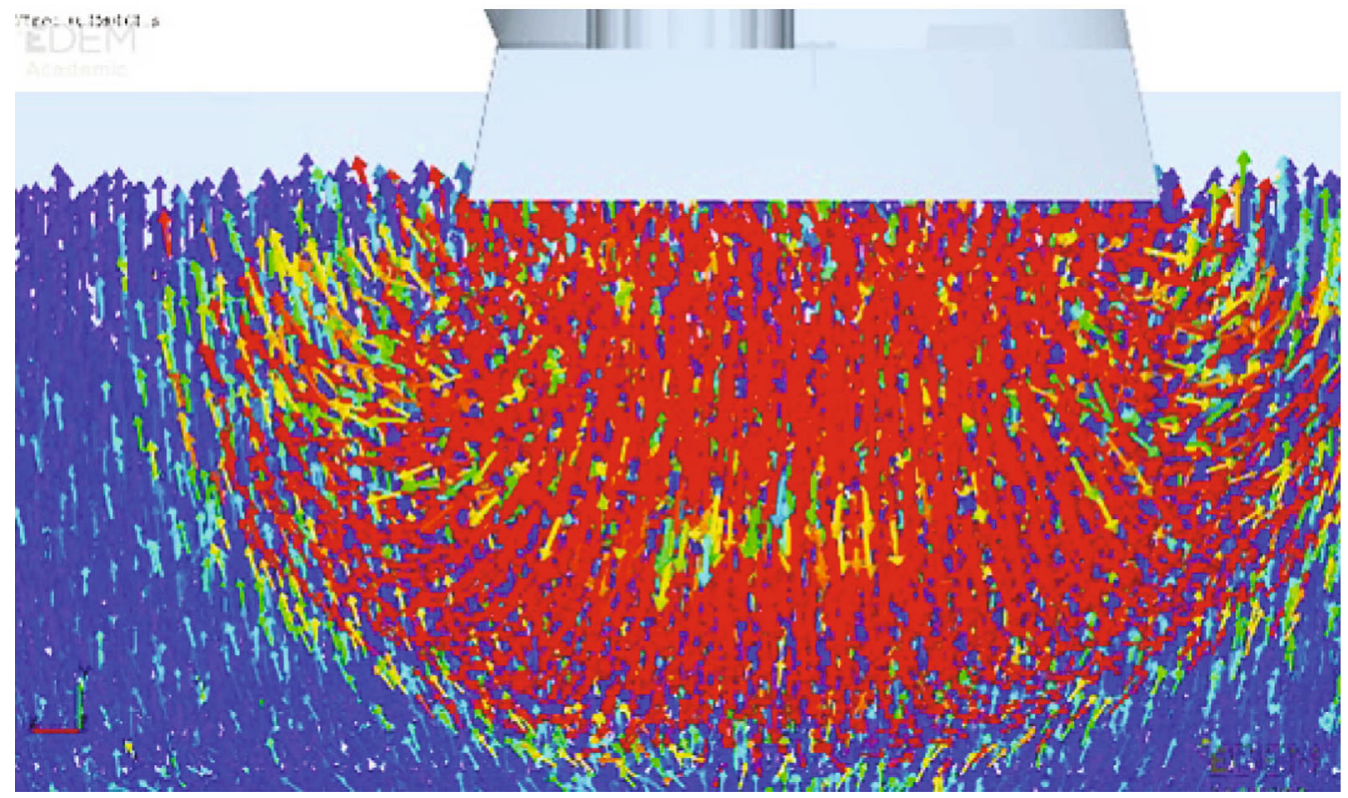

FIgURE 20: Landing impact model and lunar soil impact trajectory.

From the simulation deformation results of the square honeycomb, it can be seen that when the honeycomb is compressed in the $W$ direction, its deformation first occurs in the middle of the honeycomb core, and the compression direction is perpendicular to the compression direction. The state extends to both sides. When the honeycomb is compressed in the $L$ direction since the strength of the double-walled thick side of the honeycomb is higher than 


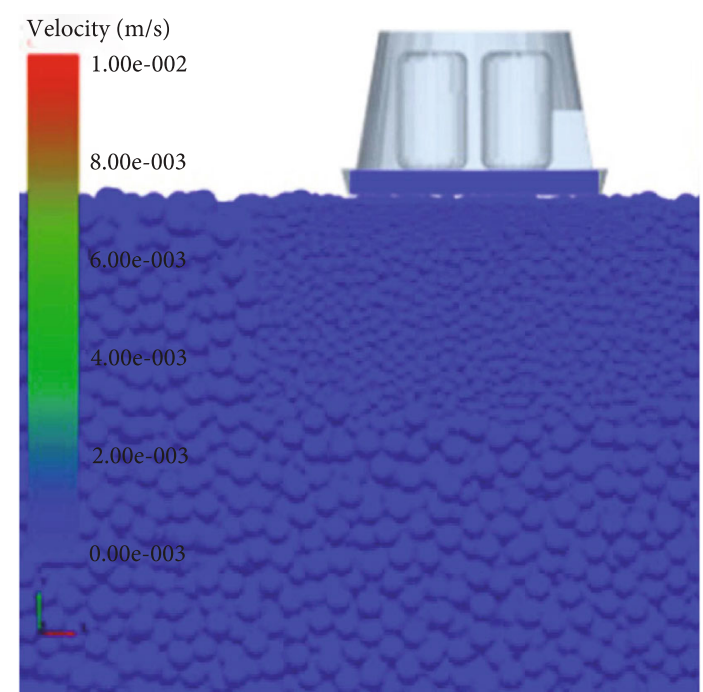

(a) Slope $0^{\circ}$

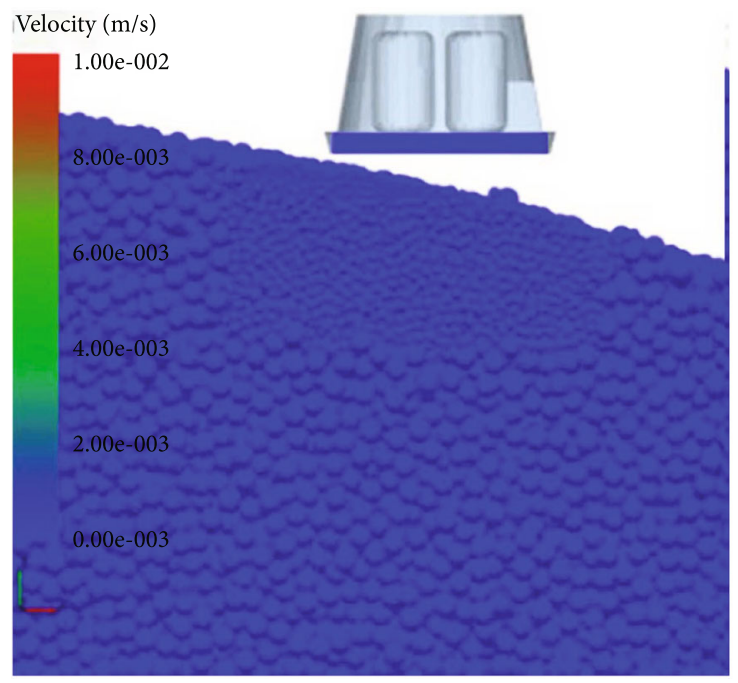

(c) Slope $10^{\circ}$

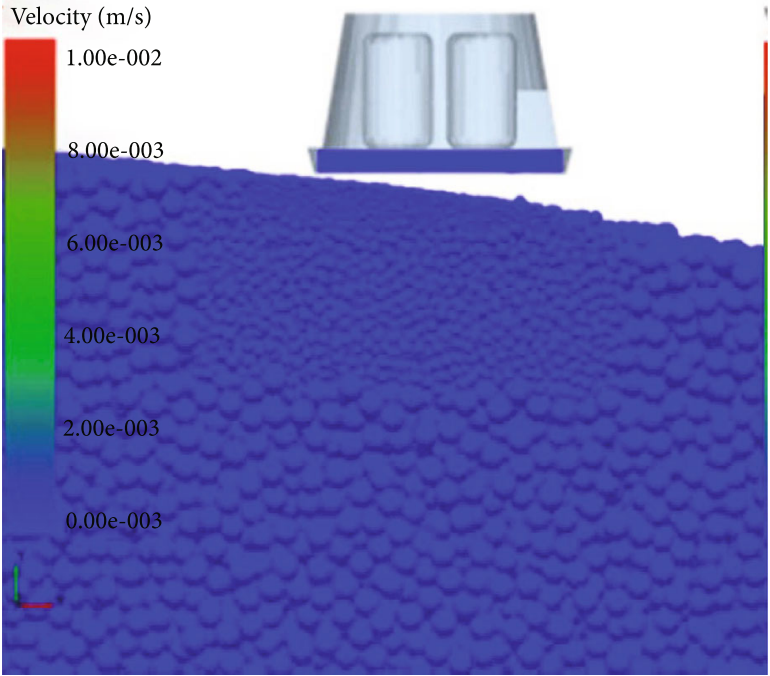

(b) Slope 5

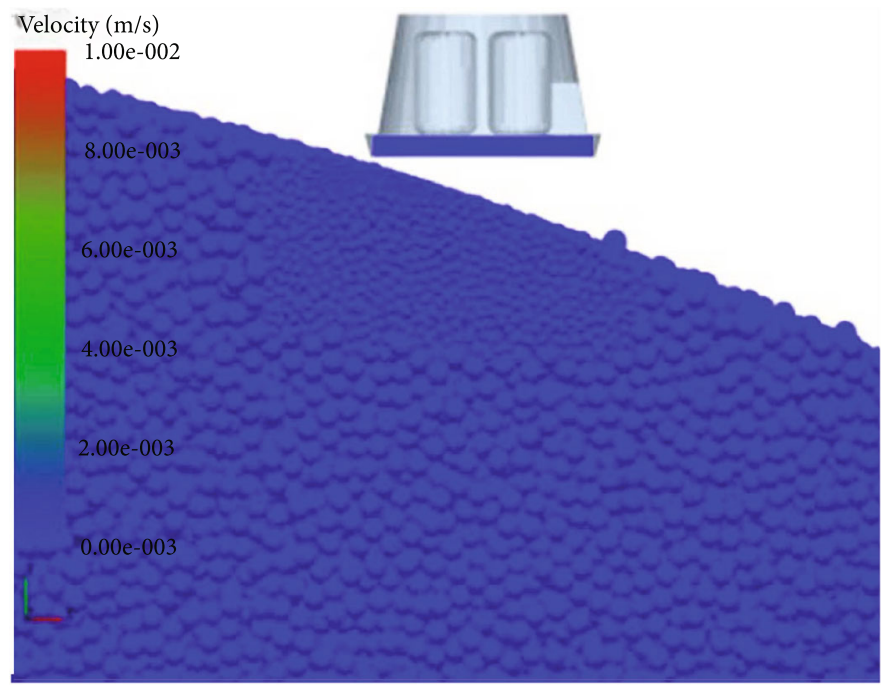

(d) Slope $15^{\circ}$

FIGURE 21: Schematic diagram of landing models under different slope conditions.

that of the single-walled thick side, its deformation mainly occurs on a single side and finally appears as the diagonal deformation of the honeycomb core. The coplanar compression deformation results of the simulated honeycomb are consistent with the experimental results of Ines et al., indicating that the discrete element honeycomb model has a good simulation effect on the honeycomb. Extract the honeycomb force during compression in the $W$ direction and $L$ direction and get the honeycomb energy absorption curve during coplanar compression according to the relationship between displacement, force, and energy, as shown in Figure 16 below. From the results, we can see that the compression during compression in the $W$ direction the force is more uniform, the energy absorption of the honeycomb rises linearly, and the collapse deformation of the platform area reaches $200 \mathrm{~mm}$. When compressed in the $L$ direction, the force of the honeycomb fluctuates greatly, and the collapse deformation of the platform area is about $170 \mathrm{~mm}$. Comparing the forces and energy absorption of the honeycomb in the $W$ direction and the $L$ direction in the coplanar compression, it can be seen that the total effective energy absorption in the $W$ direction is more, and the energy absorption effect is more stable. The experimental results are consistent with those obtained by Chuli [37].

3.2. Cobweb Honeycomb Compression Simulation Test. The discrete element model of the spider web honeycomb is composed of spherical particles, and bonding bonds are formed between the particles so that the discrete particles are accumulated and formed. The stiffness, strength, mechanical properties, and cushioning characteristics of the model are determined by the material properties and bonding bond properties of the spherical particles. The discrete element parameters of the discrete element honeycomb are shown in Table 2. The properties of the bond bonding include volume stiffness, shear stiffness, normal stress strength, shear stress strength, and bend radius. This section of the web is compressed by the integral honeycomb 


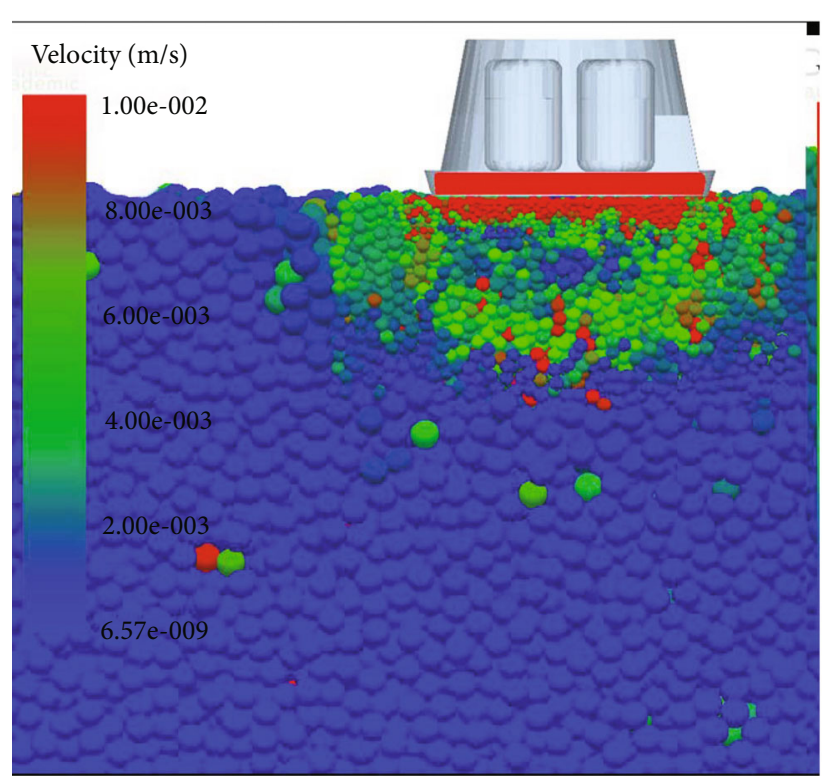

(a) Slope $0^{\circ}$

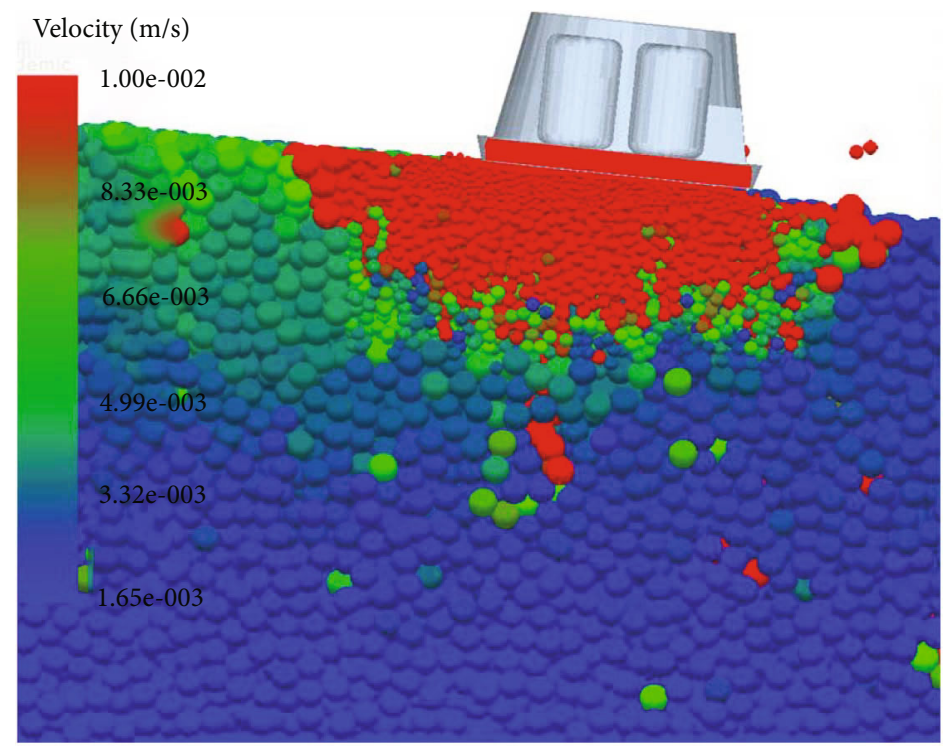

(b) Slope $5^{\circ}$

Figure 22: Continued. 


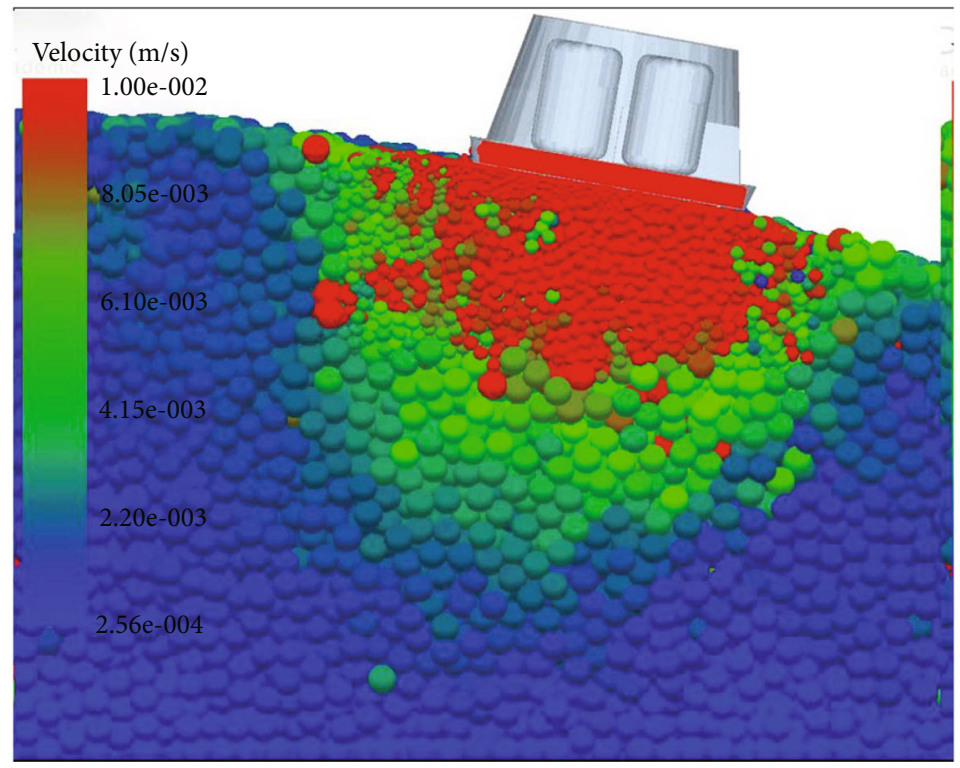

(c) Slope $10^{\circ}$

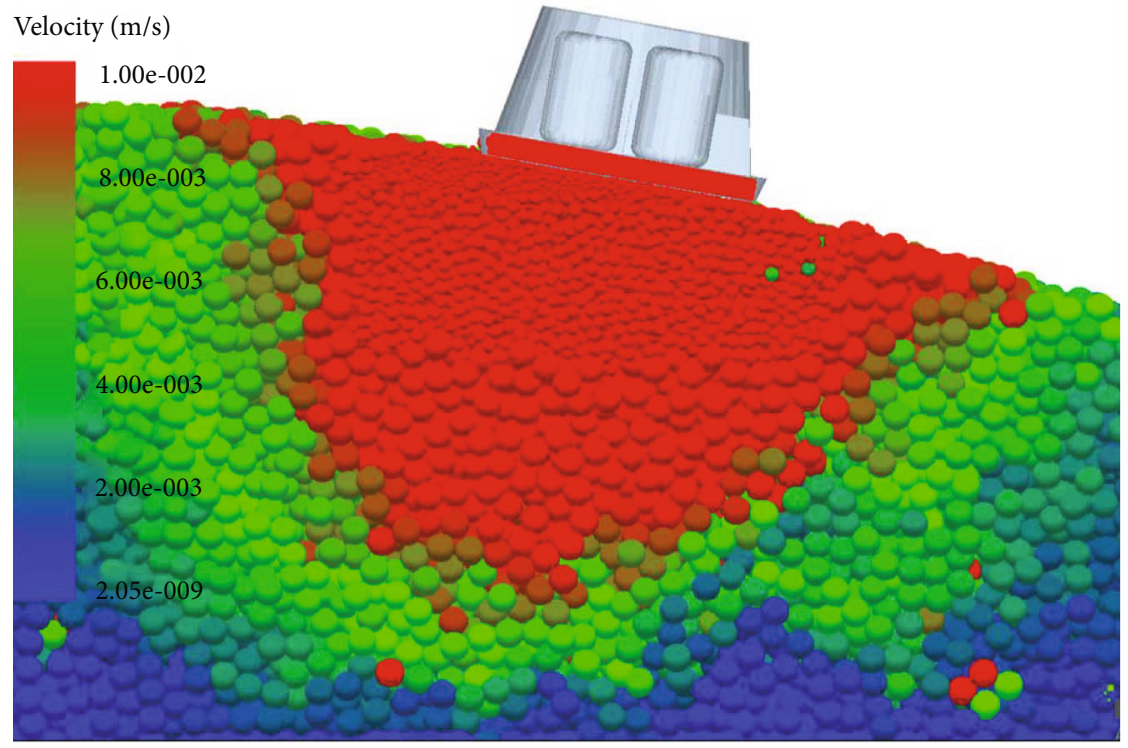

(d) Slope $15^{\circ}$

Figure 22: Simulation results of landing buffer under different slope conditions.

simulation test simulation to verify the feasibility of the model and its energy absorbing deformation to analyze the situation. Compression deformation of spider web honeycomb is shown in Figure 17 below.

It can be seen from the deformation results that the honeycomb structure maintained good flexibility during the compression process, and no particle collapse occurred, which proved the availability of the model and its parameters. In terms of deformation, the honeycomb will gradually buckle and deform in the axial direction under pressure and produce corrugated folds in the height direction. The stress is uniformly transferred throughout the spiderweb-like honeycomb and only in the part where the outermost boundary particles protrude, causing large stress.
In the EDEM postprocessing, the display mode of the model is adjusted to the bonding bond mode, and the deformation results of the honeycomb bonding bond structure before and after compression are shown in Figure 18 below.

In the early stage of compression, the internal particles are bonded to each other to form a dense and neat bonding bond. The overall appearance of the spider web radiates outwards. During the compression process, the bonding bond still maintains a good connection through the honeycomb hexagonal holes. The distortion and deformation of the upper plate absorb the compressive energy transferred from the surface of the upper platen.

3.3. Buffer Characteristics of the Whole Machine. After completing the research on the honeycomb cushioning material 


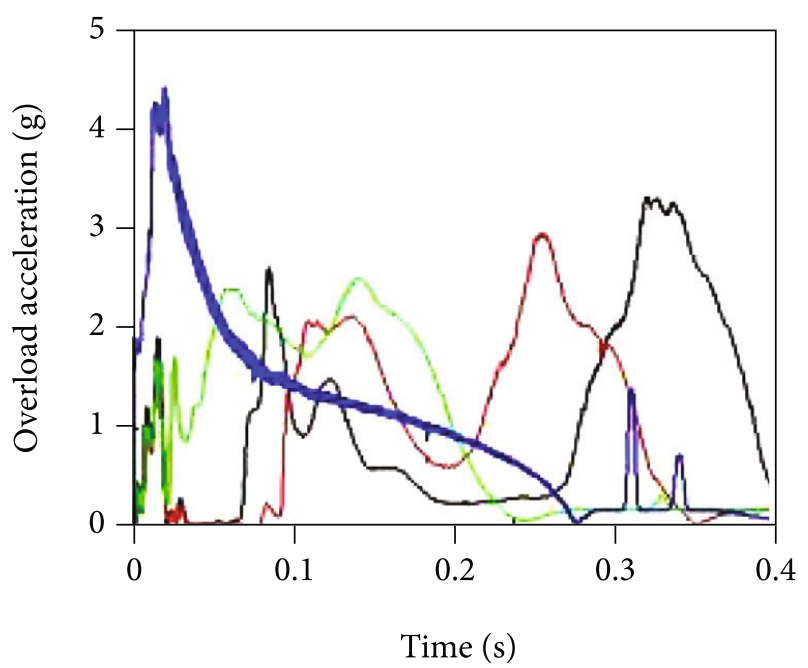

(a) Overload acceleration

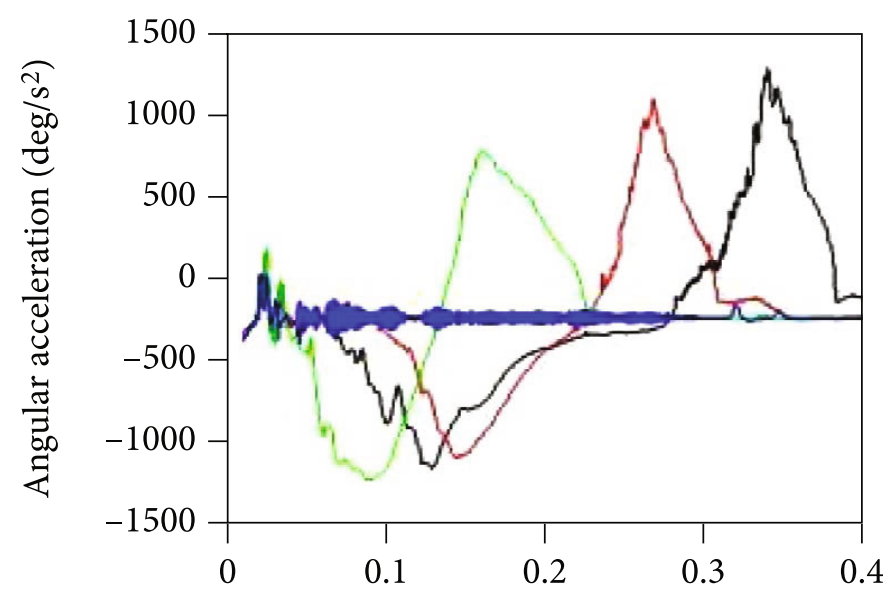

Time (s)

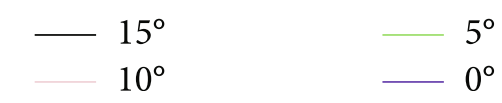

(b) Angular acceleration

FIgURE 23: Acceleration vs. time curve under different slopes.

and the spider web energy absorbing structure, it is necessary to integrate them to establish a coupled simulation model of the entire lander and study the cushioning performance of the landing process.

\subsubsection{Buffer Characteristics of the Whole Aircraft during} Landing Simulation. After building a coupled simulation platform for the entire lander, simulate its landing process and observe the landing process as shown in Figure 19. At the beginning of the simulation, to reduce the overall simulation duration, the lander is set to be in contact with the lunar surface. In ADAMS, the landing speed is directly controlled. The landing height is small, and the moon surface acceleration is small. This speed can be considered as the landing speed when it touches the moon's surface. At the $0.05 \mathrm{~s}$ position, the lander comes into contact with the lunar soil, and the lander begins to impose an impact load on the lunar soil. Under the action of the impact inertia, it pushes the lunar surface to sink and compresses the honeycomb for a cushioned landing. The combined effect of the lunar soil and the honeycomb continuously consumers its impact on kinetic energy until the lander's speed drops to zero, and finally, a slight rebound occurs in the elastic collision with the lunar soil. Under the action of gravity, it finally steadily landed on the lunar soil surface.

During the entire landing process, the surface of the internal honeycomb particles is compressed and gradually deformed by force, absorbing the kinetic energy during landing, and the surface lunar soil in contact with the lander produces bearing capacity. The impact kinetic energy of the lander is partially converted into the kinetic energy of the lunar soil. The particle movement trajectory inside the lunar soil particles is shown in Figure 20. The lunar soil velocity close to the lander moves downwards vertically. With a power exponential growth, the lunar soil begins to move diagonally upwards on both sides of the surrounding area and finally causes the particles on the lunar surface to rush out of the surface. Macroscopically, it appears as the splash of lunar soil particles, and the impact kinetic energy is directly consumed. At the same time, part of the impact energy is consumed by the friction caused by the flow and misalignment of the internal lunar soil particles.

3.3.2. The Influence of Lunar Slope on Landing Cushion Characteristics. The lunar surface is undulating, forming a large range of slopes between the highlands and the lunar sea, which is not conducive to the stable landing of the lander. Even in the lunar sea area that is most suitable for landing, its maximum slope can reach $17^{\circ}$. Because of the important effect of the slope of the lunar terrain on the landing stability, this section simulates the lunar sea environment with slopes of $0^{\circ}, 5^{\circ}, 10^{\circ}$, and $15^{\circ}$ to analyze the impact of the slope on the landing buffer characteristics. The landing models under different slope conditions are established in EDEM as shown in Figure 21. The slope surface is rotated $5^{\circ}, 10^{\circ}$, and $15^{\circ}$ clockwise around the $Z$-axis in the XZ plane, and coupled simulations are performed, respectively. The results are shown in Figure 22. The experimental methods and conclusions correspond well to the research done by Ji [38].

A summary of the lander's overload acceleration curve with time under different slopes is shown in Figure 23(a). When the landing ground is flat, its overload acceleration peak is only one, because the contact surface with the lunar soil is large, and its peak level is relatively large. It is about twice as high as when there is a slope. When landing on a slope, the overload acceleration peak appears twice, and the second peak is greater than the first. The greater the slope, the longer the time interval between the two peaks and the greater the second peak of overload acceleration. The angular acceleration curve of the lander under different slopes is shown in Figure 23(b) below. When the landing 
ground is flat, the angular acceleration is almost zero. When landing on a slope, the angular acceleration peaks appear twice. The larger the slope angle, the first peak is slightly reduced, and the second peak is larger.

\section{Conclusions}

This paper takes the lunar lander as the research object and uses discrete elements and multibody dynamics to simulate its landing buffer process. Aiming at the aluminum honeycomb buffer device of the lander, a discrete element modeling method is proposed. The mechanical properties and deformation mechanism of the discrete element honeycomb are analyzed and studied, and the feasibility of the discrete element modeling method is verified. On this basis, for a large lander, a spider web honeycomb structure is proposed, its modeling is completed through the secondary development program, and its discrete element structure is tested. Finally, the lunar soil, honeycomb, and lander are coupled, the influence of different working conditions on the landing cushion performance is studied, and the corresponding cushion prediction model is obtained.

\section{Data Availability}

The data used to support the findings of this study are available from the corresponding author upon reasonable request.

\section{Conflicts of Interest}

The authors declare that there is no conflict of interest regarding the publication of this paper.

\section{Acknowledgments}

This work was financially supported by the National Key R\&D Program of China under Grant 2019YFB1309600, the National Nature Science Foundation of China [No. 62073229, No. 51902026, No. U1637207], the Self-Planned Task of State Key Laboratory of Robot Technology and System (HIT) (No. SKLRS201801B), the Qian Xuesen Laboratory of Space Technology Seed Fund (No. QXSZZJJ03-03), and the International Science and Technology Cooperation Program of China (No. 2014DFR50250).

\section{References}

[1] C. Wang, Z. Deng, H. Gao, and R. Liu, "Research status of lunar landers at home and abroad," Missile and Space Launch Technology, vol. 3, no. 4, 2006.

[2] Z. Deng and S. Wang, "Experimental study on cushioning performance of three-supported lunar lander," Journal of Harbin Institute of Technology, vol. 34, no. 1, pp. 32-34, 2007.

[3] S. Wang and Z. Deng, Impact performance test of damping material for lunar lander, no. 1, 2007Missile and Space Vehicle Technology, 2007.

[4] D. Li, "On the moon resources and space moon exploration," Space Return and Remote Sensing, vol. 25, no. 1, pp. 60-64, 2004.
[5] H. Liu, Future new energy-lunar helium-3, China Electric Power Education, 2014.

[6] Z. Ouyang, C. Li, Y. Zou, and J. Liu, "The progress of lunar exploration and my country's lunar exploration," Chinese Science Foundation, vol. 17, no. 4, pp. 193-197, 2003.

[7] L. J. Gibson and M. F. Ashby, Cellular Solids: Structure and Properties, Cambridge university press, 1999.

[8] R. K. McFarland Jr., "Hexagonal cell structures under postbuckling axial load," AIAA Journal, vol. 1, no. 6, pp. 13801385, 1963.

[9] J. Zhang and M. F. Ashby, "The out-of-plane properties of honeycombs," International Journal of Mechanical Sciences, vol. 34, no. 6, pp. 475-489, 1992.

[10] W. E. Baker, T. C. Togami, and J. C. Weydert, "Static and dynamic properties of high-density metal honeycombs," International Journal of Impact Engineering, vol. 21, no. 3, pp. 149$163,1998$.

[11] S. T. Hong, J. Pan, T. Tyan, and P. Prasad, "Quasi-static crush behavior of aluminum honeycomb specimens under compression dominant combined loads," International Journal of Plasticity, vol. 22, no. 1, pp. 73-109, 2006.

[12] X. Zhang, H. Zhang, and Z. Wen, "Experimental and numerical studies on the crush resistance of aluminum honeycombs with various cell configurations," International Journal of Impact Engineering, vol. 66, pp. 48-59, 2014.

[13] L. Aktay, A. F. Johnson, and B. H. Kröplin, "Numerical modelling of honeycomb core crush behaviour," Engineering Fracture Mechanics, vol. 75, no. 9, pp. 2616-2630, 2008.

[14] J. Huang, J. Man, J. Yang, Y. Ye, R. Liu, and Z. Deng, “Experimental study on the adaptability of aluminum honeycomb materials to temperature environment," Manned Spaceflight, vol. 22, no. 3, pp. 313-316, 2016.

[15] Y. Zhang and Z. Wang, "Research on the crashworthiness of honeycomb Sandwich panels," Journal of Jiangsu University of Science and Technology (Natural Science Edition), vol. 21, no. 3, pp. 1-5, 2007.

[16] X. Zhang, Y. Liu, and Z. Zhang, "Research on the in-plane impact properties of composite honeycomb materials," Engineering Mechanics, vol. 26, no. 6, pp. 220-225, 2009.

[17] P. M. Quoc, D. Krzikalla, J. Mesicek et al., “On Aluminum honeycomb impact attenuator designs for formula student competitions," Symmetry, vol. 12, no. 10, p. 1647, 2020.

[18] B. Mertani, B. Keskes, and M. Tarfaoui, "Experimental analysis of the crushing of honeycomb cores under compression," Journal of Materials Engineering and Performance, vol. 28, no. 3, pp. 1628-1638, 2019.

[19] Y. Xing, S. Yang, S. Lu, Y. An, E. Zhao, and J. Zhai, "Energy absorption and optimization of bi-directional corrugated honeycomb aluminum," Composites Part B: Engineering, vol. 219, p. 108914, 2021.

[20] J. Z. Yang, W. Zhu, J. F. Man, and F. M. Zeng, "Design and verification of the landing impact attenuation system for Chang\&rsquo;E-3 lander," Science in China: Technological Sciences, vol. 44, no. 5, pp. 440-449, 2014.

[21] H. Han, Y. Wang, Y. Jiang, and H. lou, "Features and applications of foreign deep space probe landing buffer systems," Spacecraft Engineering, vol. 21, no. 6, pp. 7-24, 2012.

[22] J. Chen, H. Nie, H. Bai, and Q. Zhao, "Overview of the development of the soft landing buffer mechanism of the lunar probe," in Proceedings of the Third Academic Conference of the Deep Space Exploration Technology Professional Committee 
of the Chinese Astronautical Society, Northwest University of Technology, Xi'an, China, 2006.

[23] A. L. Joo and C. John, Lunar Lander Conceptual Design, NASA, 1989.

[24] J. Yang, F. Zeng, J. Man, and H. Lou, Research on the characteristics of broach type and expansion ring seat buffer schemes, Academic Seminar of the Aircraft General Professional Committee of the Chinese Academy of Astronautics, 2005.

[25] H. Li, Theoretical and Application Research of Magnetorheological Buffer Damper in the Soft Landing Process of Lunar Lander, Northwestern Polytechnical University, 2002.

[26] Y. Dong, T. Yu, and Z. Zhang, "Mechanical design and energy absorption analysis of spherical honeycomb core for softlanding device buffer shell," Acta Mechanica, vol. 231, no. 10, pp. 4243-4257, 2020.

[27] L. Zhu, Liu, Guo, Xu, Hou, and Niu, "Modeling and analysis of an electromagnetic fully variable valve train with a magnetorheological buffer," Electronics, vol. 8, no. 9, p. 996, 2019.

[28] G. Aravind, S. Vishnu, K. V. Amarnath et al., "Design, analysis and stability testing of lunar lander for soft-landing," Materials Today: Proceedings, vol. 24, pp. 1235-1243, 2020.

[29] Y. Zhou, H. Zhao, M. Chen, J. Tu, and L. Yan, “Automatic detection of lunar craters based on DEM data with the terrain analysis method," Planetary and Space Science, vol. 160, pp. 1-11, 2018.

[30] Z. Jiang, Research on the Interaction between Lander Foot Pads and Lunar Soil, Zhejiang University, 2013.

[31] S. Fan, J. Yang, S. Xiang, and C. Liu, "Preliminary study on simulation of lunar terrain and landform environment," Spacecraft Environmental Engineering, vol. 24, no. 1, pp. 1520, 2007.

[32] S. Zhong, Simulated Lunar Soil Mechanical Properties and Soft Landing Foot Pad Dynamic Response Research, Zhejiang University, 2012.

[33] Y. Zheng, O. Ziyuan, S. Wang, and Y. Zhou, "Physical and mechanical properties of lunar soil," Minerals and Rocks, vol. 24, no. 4, pp. 14-19, 2004.

[34] S. Zhang, W. Chen, D. Gao, L. Xiao, and L. Han, "Experimental study on dynamic compression mechanical properties of aluminum honeycomb structures," Applied Sciences, vol. 10, no. 3, p. 1188, 2020.

[35] I. Ivañez, L. M. Fernandez-Cañadas, and S. Sanchez-Saez, "Compressive deformation and energy-absorption capability of aluminium honeycomb core," Composite Structures, vol. 174, pp. 123-133, 2017.

[36] K. Tanaka, M. Nishida, K. Tomita, and T. Hayakawa, Strain characteristics of aluminum honeycombs under the static and impact compressions, Springer, Berlin Heidelberg, 2009.

[37] A. J. Chuli and M. R. Said, "Experimental investigation on empty aluminium honeycomb under quasi-static lateral compression," Proceedings of Mechanical Engineering Research Day, vol. 2016, pp. 139-140, 2016.

[38] S. Ji and S. Liang, "DEM-FEM-MBD coupling analysis of landing process of lunar lander considering landing mode and buffering mechanism," Advances in Space Research, vol. 68, no. 3, pp. 1627-1643, 2021. 